\title{
Interventions for eye movement disorders due to acquired brain injury (Review)
}

Rowe FJ, Hanna K, Evans JR, Noonan CP, Garcia-Finana M, Dodridge CS, Howard C, Jarvis KA, MacDiarmid SL, Maan T, North L, Rodgers H

Rowe FJ, Hanna K, Evans JR, Noonan CP, Garcia-Finana M, Dodridge CS, Howard C, Jarvis KA, MacDiarmid SL, Maan T, North L, Rodgers $\mathrm{H}$.

Interventions for eye movement disorders due to acquired brain injury.

Cochrane Database of Systematic Reviews 2018, Issue 3. Art. No.: CD011290.

DOI: 10.1002/14651858.CD011290.pub2.

www.cochranelibrary.com 
TABLE OF CONTENTS

ABSTRACT 1

PLAIN LANGUAGE SUMMARY

SUMMARY OF FINDINGS

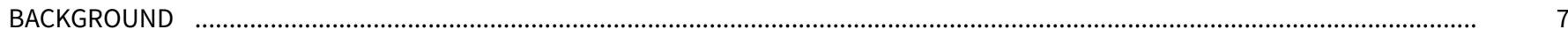

OBJECTIVES

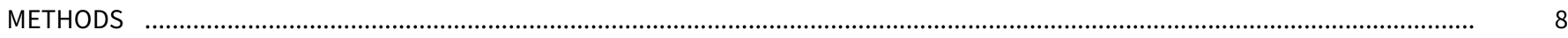

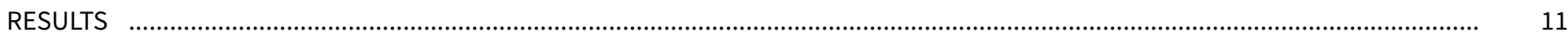

Figure 1. . .

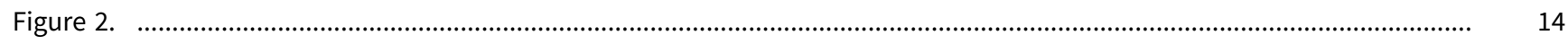

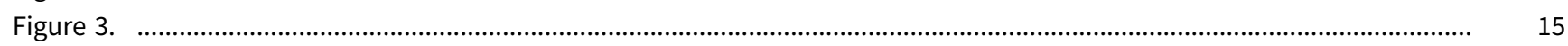

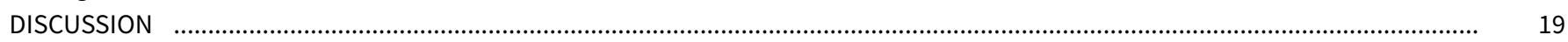

AUTHORS' CONCLUSIONS

ACKNOWLEDGEMENTS

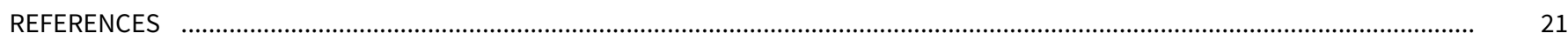

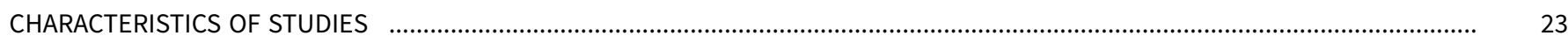

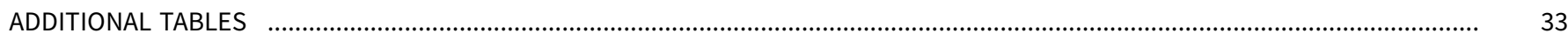

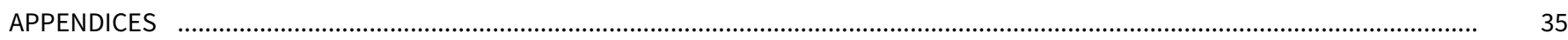

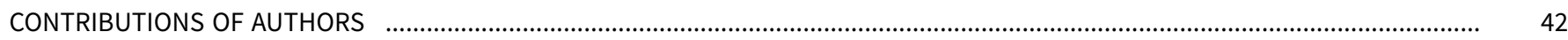

DECLARATIONS OF INTEREST

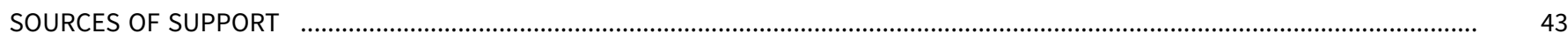

DIFFERENCES BETWEEN PROTOCOL AND REVIEW

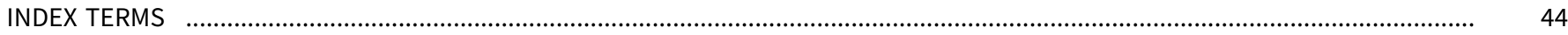


[Intervention Review]

\section{Interventions for eye movement disorders due to acquired brain injury}

Fiona J Rowe1, Kerry Hanna1, Jennifer R Evans², Carmel P Noonan³, Marta Garcia-Finana4 , Caroline S Dodridge5, Claire Howard6, Kathryn A Jarvis7 7 Sonia L MacDiarmid8, Tallat Maan 9 , Lorraine North10, Helen Rodgers 11

1Department of Health Services Research, University of Liverpool, Liverpool, UK. ${ }^{2}$ Cochrane Eyes and Vision, ICEH, London School of Hygiene \& Tropical Medicine, London, UK. 3Department of Ophthalmology, Aintree University Hospitals NHS Foundation Trust, Liverpool, UK. ${ }^{4}$ Biostatistics, University of Liverpool, Liverpool, UK. ${ }^{5}$ Orthoptics, Oxford University Hospitals NHS Trust, Oxford, UK. 60rthoptics, Salford Royal NHS Foundation Trust, Manchester, UK. ${ }^{7}$ Occupational Therapy, University of Liverpool, Liverpool, UK. 8Department of Orthoptics, Warrington and Halton Hospitals NHS Foundation Trust, Warrington, UK. 9'Community Eye Service, Pennine Care NHS Foundation Trust, Ashton-under-Lyne, UK. ${ }^{10}$ Orthoptics, Frimley Park NHS Foundation Trust, Frimley, UK. ${ }^{11}$ Institute of Neuroscience, Newcastle University, Newcastle, UK

Contact: Fiona J Rowe, Department of Health Services Research, University of Liverpool, Waterhouse Building (B211), 1-3 Brownlow Street, Liverpool, L69 3GL, UK. rowef@liverpool.ac.uk.

Editorial group: Cochrane Eyes and Vision Group.

Publication status and date: New, published in Issue 3, 2018.

Citation: Rowe FJ, Hanna K, Evans JR, Noonan CP, Garcia-Finana M, Dodridge CS, Howard C, Jarvis KA, MacDiarmid SL, Maan T, North L, Rodgers $\mathrm{H}$. Interventions for eye movement disorders due to acquired brain injury. Cochrane Database of Systematic Reviews 2018, Issue 3. Art. No.: CD011290. DOI: 10.1002/14651858.CD011290.pub2.

Copyright $@ 2018$ The Cochrane Collaboration. Published by John Wiley \& Sons, Ltd.

\section{A B S T R A C T}

\section{Background}

Acquired brain injury can cause eye movement disorders which may include: strabismus, gaze deficits and nystagmus, causing visual symptoms of double, blurred or 'juddery' vision and reading difficulties. A wide range of interventions exist that have potential to alleviate or ameliorate these symptoms. There is a need to evaluate the effectiveness of these interventions and the timing of their implementation.

\section{Objectives}

We aimed to assess the effectiveness of any intervention and determine the effect of timing of intervention in the treatment of strabismus, gaze deficits and nystagmus due to acquired brain injury. We considered restitutive, substitutive, compensatory or pharmacological interventions separately and compared them to control, placebo, alternative treatment or no treatment for improving ocular alignment or motility (or both).

\section{Search methods}

We searched the Cochrane Central Register of Controlled Trials (CENTRAL) (containing the Cochrane Eyes and Vision Trials Register) (2017, Issue 5), MEDLINE Ovid, Embase Ovid, CINAHL EBSCO, AMED Ovid, PsycINFO Ovid, Dissertations \& Theses (PQDT) database, PsycBITE (Psychological Database for Brain Impairment Treatment Efficacy), ISRCTN registry, ClinicalTrials.gov, Health Services Research Projects in Progress (HSRProj), National Eye Institute Clinical Studies Database and the World Health Organization (WHO) International Clinical Trials Registry Platform (ICTRP). The databases were last searched on 26 June 2017. No date or language restrictions were used in the electronic searches for trials. We manually searched the Australian Orthoptic Journal, British and Irish Orthoptic Journal, and ESA, ISA and IOA conference proceedings. We contacted researchers active in this field for information about further published or unpublished studies.

\section{Selection criteria}

We included randomised controlled trials (RCTs) of any intervention for ocular alignment or motility deficits (or both) due to acquired brain injury. 


\section{Data collection and analysis}

Two review authors independently selected studies and extracted data. We used standard methods expected by Cochrane. We employed the GRADE approach to interpret findings and assess the quality of the evidence.

\section{Main results}

We found five RCTs (116 participants) that were eligible for inclusion. These trials included conditions of acquired nystagmus, sixth cranial nerve palsy and traumatic brain injury-induced ocular motility defects. We did not identify any relevant studies of restitutive interventions.

We identified one UK-based trial of a substitutive intervention, in which botulinum toxin was compared with observation in 47 people with acute sixth nerve palsy. At four months after entry into the trial, people given botulinum toxin were more likely to make a full recovery (reduction in angle of deviation within 10 prism dioptres), compared with observation (risk ratio $1.19,95 \% \mathrm{Cl} 0.96$ to 1.48 ; low-certainty evidence). These same participants also achieved binocular single vision. In the injection group only, there were 2 cases of transient ptosis out of 22 participants (9\%), and 4 participants out of $22(18 \%)$ with transient vertical deviation; a total complication rate of $24 \%$ per injection and $27 \%$ per participant. All adverse events recovered. We judged the certainty of evidence as low, downgrading for risk of bias and imprecision. It was not possible to mask investigators or participants to allocation, and the follow-up between groups varied.

We identified one USA-based cross-over trial of a compensatory intervention. Oculomotor rehabilitation was compared with sham training in 12 people with mild traumatic brain injury, at least one year after the injury. We judged the evidence from this study to be very lowcertainty. The study was small, data for the sham training group were not fully reported, and it was unclear if a cross-over study design was appropriate as this is an intervention with potential to have a permanent effect.

We identified three cross-over studies of pharmacological interventions for acquired nystagmus, which took place in Germany and the USA. These studies investigated two classes of pharmacological interventions: GABAergic drugs (gabapentin, baclofen) and aminopyridines (4aminopyridines (AP), 3,4-diaminopyridine (DAP)). We judged the evidence from all three studies as very low-certainty because of small numbers of participants (which led to imprecision) and risk of bias (they were cross-over studies which did not report data in a way that permitted estimation of effect size).

One study compared gabapentin (up to $900 \mathrm{mg} / \mathrm{day}$ ) with baclofen (up to $30 \mathrm{mg} / \mathrm{day}$ ) in 21 people with pendular and jerk nystagmus. The follow-up period was two weeks. This study provides very low-certainty evidence that gabapentin may work better than baclofen in improving ocular motility and reducing participant-reported symptoms (oscillopsia). These effects may be different in pendular and jerk nystagmus, but without formal subgroup analysis it is unclear if the difference between the two types of nystagmus was chance finding. Quality of life was not reported. Ten participants with pendular nystagmus chose to continue treatment with gabapentin, and one with baclofen. Two participants with jerk nystagmus chose to continue treatment with gabapentin, and one with baclofen. Drug intolerance was reported in one person receiving gabapentin and in four participants receiving baclofen. Increased ataxia was reported in three participants receiving gabapentin and two participants receiving baclofen.

One study compared a single dose of 3,4-DAP $(20 \mathrm{mg})$ with placebo in 17 people with downbeat nystagmus. Assessments were made 30 minutes after taking the drug. This study provides very low-certainty evidence that 3,4-DAP may reduce the mean peak slow-phase velocity, with less oscillopsia, in people with downbeat nystagmus. Three participants reported transient side effects of minor perioral/ distal paraesthesia.

One study compared a single dose of 4-AP with a single dose of 3,4-DAP (both $10 \mathrm{mg}$ doses) in eight people with downbeat nystagmus. Assessments were made 45 and 90 minutes after drug administration. This study provides very low-certainty evidence that both 3,4-DAP and 4-AP may reduce the mean slow-phase velocity in people with downbeat nystagmus. This effect may be stronger with 4-AP.

\section{Authors' conclusions}

The included studies provide insufficient evidence to inform decisions about treatments specifically for eye movement disorders that occur following acquired brain injury. No information was obtained on the cost of treatment or measures of participant satisfaction relating to treatment options and effectiveness. It was possible to describe the outcome of treatment in each trial and ascertain the occurrence of adverse events.

\section{PLAIN LANGUAGE SUMMARY}

\section{Interventions for eye movement disorders due to acquired brain injury}

\section{What is the aim of this review?}

The aim of this Cochrane Review was to find out what treatments work well to improve eye position and eye movement disorders due to acquired brain injury, and when is the best time to use them.

\section{Key messages}

The evidence on the benefits and harms of treatments for eye movement disorders due to acquired brain injury is currently very lowcertainty. 


\section{What was studied in this review?}

Acquired brain injury is any injury that occurs after birth and causes damage to the brain's function. Strabismus is a condition in which the eyes are out of alignment, with one or both eyes turned in, out, up or down. Ocular motility (eye movement) disorders are defects that prevent normal movement of the eyes. Nystagmus is a condition where the eye movements are not steady and, instead, the eyes wobble. Treatment options include eye therapy, glasses, prisms, occlusion, botulinum toxin or surgery, to reduce the deviation or movement of the eyes. Currently there are no clear recommendations on when is best to provide these treatments, how much these treatments cost and whether treatments are of benefit to people with eye alignment and movement disorders occurring after acquired brain injury.

\section{What are the main results of this review?}

Cochrane researchers found five relevant studies with a total of 116 participants. One study was from the UK and looked at botulinum toxin compared to observation in people with recent onset sixth nerve palsy. One study from the USA compared eye movement training with sham (false) training in people with mild traumatic brain injury. Three studies took place in Germany or the USA and compared medical drug treatments in people with acquired nystagmus. The review provides:

- low-certainty evidence that people with sixth nerve palsy may have a slightly better chance of a reduction in visual symptoms when given botulinum toxin compared with no treatment.

- very low-certainty evidence on eye movement treatment for people with brain injury due to trauma and use of medical drugs for nystagmus, where treatments show slightly better improved symptoms.

\section{How up-to-date is this review?}

Cochrane researchers searched for studies that had been published up to 26 June 2017. 


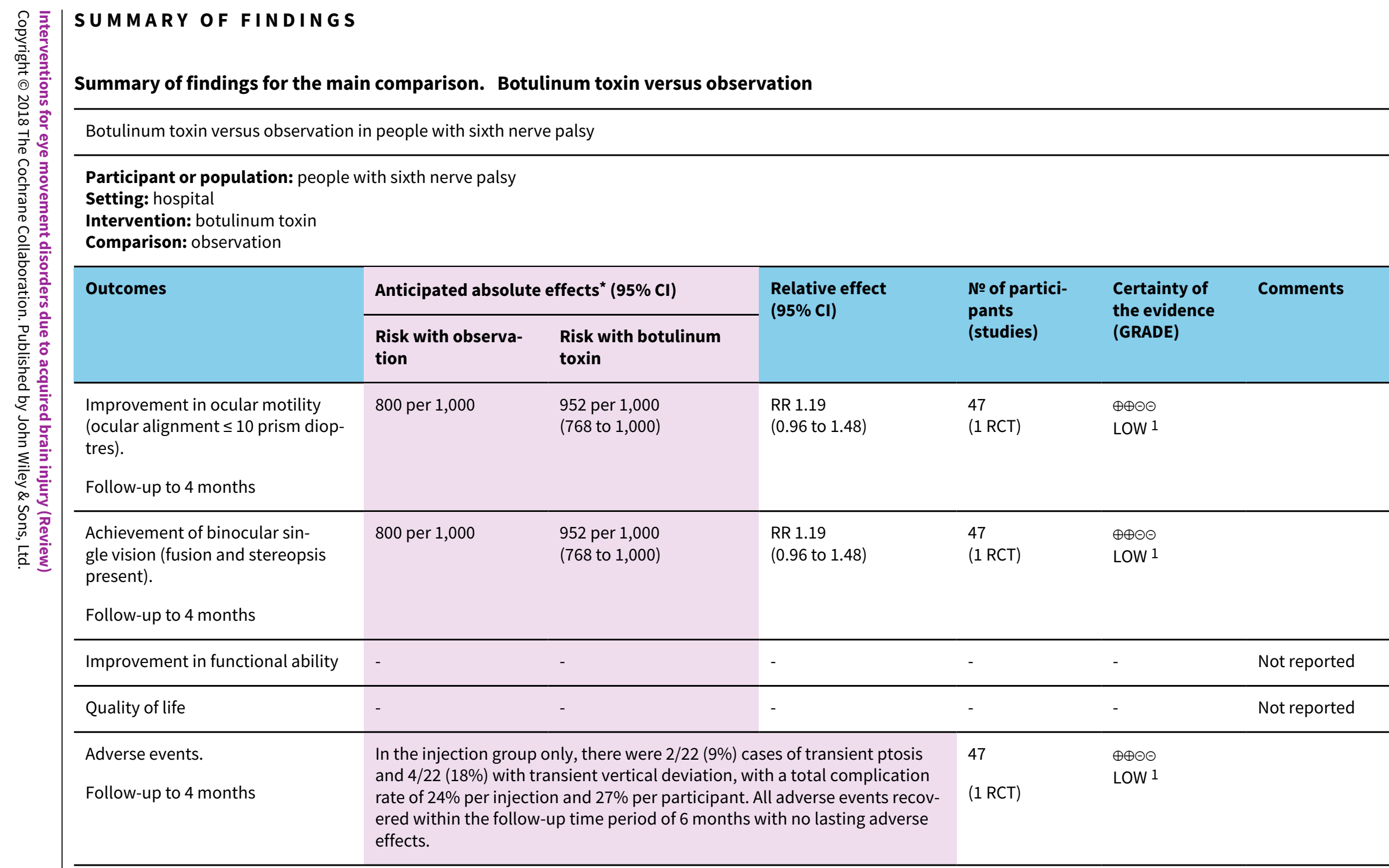

${ }^{\star}$ The risk in the intervention group (and its $95 \%$ confidence interval) is based on the assumed risk in the comparison group and the relative effect of the intervention (and its $95 \% \mathrm{Cl})$.

Cl: Confidence interval; RR: Risk ratio

\section{GRADE Working Group grades of evidence}

High-certainty: We are very confident that the true effect lies close to that of the estimate of the effect 
Moderate-certainty: We are moderately confident in the effect estimate: The true effect is likely to be close to the estimate of the effect, but there is a possibility that it is substantially different

Low-certainty: Our confidence in the effect estimate is limited: The true effect may be substantially different from the estimate of the effect

Very low-certainty: We have very little confidence in the effect estimate: The true effect is likely to be substantially different from the estimate of effect

1 Downgraded one level for risk of bias (investigators were aware of the randomisation and it was not possible to mask investigators or participants to the allocation and there was variable follow-up between groups) and downgraded one level for imprecision (confidence intervals include 1, no effect).

\section{Summary of findings 2 . Pharmacological treatment}

Pharmacological treatments (Gabapentin / Baclofen / 3,4-DAP / 4-AP) for people with acquired nystagmus

Participant or population: people with acquired nystagmus

Setting: eye clinic

Intervention: pharmacological treatment

Comparison: placebo or other drugs

\begin{tabular}{|c|c|c|c|}
\hline Comparison & Main findings & $\begin{array}{l}\text { № of participants } \\
\text { (studies) }\end{array}$ & $\begin{array}{l}\text { Certainty of the evidence } \\
\text { (GRADE) }\end{array}$ \\
\hline $\begin{array}{l}\text { Gabapentin up to } 900 \mathrm{mg} / \\
\text { day) versus baclofen (up } \\
\text { to } 30 \mathrm{mg} / \text { day). } \\
\text { Follow-up } 2 \text { weeks }\end{array}$ & $\begin{array}{l}\text { Gabapentin may work better than baclofen in improving ocular motility and re- } \\
\text { ducing participant-reported symptoms (oscillopsia). These effects may be dif- } \\
\text { ferent in pendular and jerk nystagmus but there was no formal subgroup analy- } \\
\text { sis so it is unclear if the difference between the two types of nystagmus was a } \\
\text { chance finding. Quality of life was not reported but ten participants with pendu- } \\
\text { lar nystagmus chose to continue treatment with gabapentin and one with ba- } \\
\text { clofen. Two participants with jerk nystagmus chose to continue treatment with } \\
\text { gabapentin and one with baclofen. Drug intolerance was reported in one person } \\
\text { for gabapentin and four participants for baclofen. Increased ataxia was reported in } \\
\text { three participants for gabapentin and two participants for baclofen. }\end{array}$ & $\begin{array}{l}21 \\
(1 \mathrm{RCT})\end{array}$ & $\begin{array}{l}\oplus \ominus \ominus \ominus \\
\text { VERY LOW } 1\end{array}$ \\
\hline $\begin{array}{l}\text { 3,4-DAP ( } 20 \text { mg, single } \\
\text { dose) versus placebo. } \\
\text { Assessments made } 30 \\
\text { minutes after taking the } \\
\text { drug or placebo }\end{array}$ & $\begin{array}{l}\text { 3,4-DAP may reduce the mean peak slow-phase velocity in people with downbeat } \\
\text { nystagmus. In } 10 \text { of the } 17 \text { participants, mean peak slow-phase velocity decreased } \\
\text { by more than } 50 \% \text { and these } 10 \text { people reported having less oscillopsia. No signifi- } \\
\text { cant adverse events were reported. Nine participants continued treatment. Three } \\
\text { participants reported transient side effects of minor perioral/distal paraesthesia. }\end{array}$ & $\begin{array}{l}17 \\
(1 \mathrm{RCT})\end{array}$ & $\begin{array}{l}\oplus \ominus \ominus \ominus \\
\text { VERY LOW1 }\end{array}$ \\
\hline $\begin{array}{l}\text { 4-AP ( } 10 \mathrm{mg} \text {, single dose) } \\
\text { versus 3,4-DAP (10 mg, } \\
\text { single dose) }\end{array}$ & $\begin{array}{l}\text { 3,4 DAP and 4-AP may reduce mean slow-phase velocity in people with downbeat } \\
\text { nystagmus. This effect may be stronger with 4-AP. All participants reported mild } \\
\text { paraesthesias with both medications. }\end{array}$ & $\begin{array}{l}8 \\
(1 \mathrm{RCT})\end{array}$ & $\begin{array}{l}\oplus \ominus \ominus \ominus \\
\text { VERY LOW1 }\end{array}$ \\
\hline
\end{tabular}




\section{GRADE Working Group grades of evidence}

High-certainty: We are very confident that the true effect lies close to that of the estimate of the effect

Moderate-certainty: We are moderately confident in the effect estimate: The true effect is likely to be close to the estimate of the effect, but there is a possibility that it is substantially different

Low-certainty: Our confidence in the effect estimate is limited: The true effect may be substantially different from the estimate of the effect

Very low-certainty: We have very little confidence in the effect estimate: The true effect is likely to be substantially different from the estimate of effect

1 Downgraded two levels for imprecision (due to small number of participants) and one level for serious risk of bias (cross-over study with analysis that did not permit estimation of effect size). 


\section{B A C K G R O U N D}

\section{Description of the condition}

Acquired brain injury is brain damage caused by events occurring after birth that result in permanent or temporary changes in cognition, physical, emotional and behavioural function. This may be due to trauma, surgery, stroke, brain tumour, infection, inflammation and ischaemia. Eye movement disorders following acquired brain injury may include strabismus, gaze deficits and nystagmus (Rowe 2003). In acquired brain injury, these eye movement disorders are caused by damage to the cranial nerves that supply the extra ocular muscles or to damage of the neurological areas that contribute to the control of eye movements (Pierrot-Deseilligny 2011). The incidence of visual problems in acquired brain injury is unknown but estimates are available for certain types of acquired brain injury. For example, over half of stroke survivors have visual impairments (Freeman 1988), and up to $68 \%$ of stroke survivors with visual symptoms have eye movement disorders (Rowe 2009). These impact on daily life by causing a range of difficulties including inability to maintain normal ocular alignment or move the eyes appropriately (Hepworth 2016; Jones 2006; Pedersen 1981; Rowe 2011a). Functional disabilities occur including loss of depth perception, reduced hand-eye coordination and reading impairment (Hepworth 2016; Maclntosh 2003; Rowe 2011b), and these may impede the effectiveness of rehabilitation therapy in regaining mobility, activities of daily living and quality of life (Ciuffreda 2007; MacIntosh 2003).

The true incidence of eye movement disorders in acquired brain injury is unknown and the prevalence varies depending on the cause of brain injury and area of brain involved. Strabismus is a deviation of the ocular alignment where one eye turns and which may be intermittent or constant (Fowler 1996; Rowe 2010). Strabismus occurs in a number of forms including: esotropia (in-turning deviation), exotropia (out-turning deviation) or less commonly, hypertropia (up-turning deviation), hypotropia (downturning deviation) and cyclotropia (rotatory deviation). Gaze deficits include disorders of the eye movement systems which involve saccades (fast movements of the eyes), smooth pursuits (slow, tracking movements of the eyes), vergence (opposite movements of the eyes such as convergence where both eyes turn inwards symmetrically or divergence where both eyes turn outwards), cranial nerve palsy (impairment of III, IV or VI nerve function causing abnormal eye movement and strabismus in the affected eye), bilateral gaze palsy (impaired horizontal, vertical or combined movements in both eyes), unilateral gaze palsy (internuclear ophthalmoplegia, one and a half syndrome) and vestibulo-ocular and opto-kinetic reflexes (involuntary movements of the eyes in response to moving objects and movement of the head and body) (Pedersen 1981; Rowe 2011a; Rowe 2013a). Nystagmus is a condition in which there are frequent involuntary oscillations of the eyes that result in reduced visual function (Rowe 2008).

Symptoms of eye movement disorders can include blurring of vision, diplopia (double vision), impaired depth (3-dimensional) perception, wobbling and jumbling of images, and reading difficulty (Rowe 2013b).

\section{Description of the intervention}

There are various treatments associated with strabismus, eye movement disorders and nystagmus. Primarily, treatment is directed at aligning the visual axes and improving the movement of the eyes.

Treatments for eye movement disorders can be described as restitution, compensation, substitution or pharmacology (Kerkhoff 2000; Pollock 2011). Restitutive treatment aims to restore visual function to normal; compensatory treatments aims to aid adaptation to the persistent visual impairment and substitutive treatments aim to use optical or medical aids to enhance visual function. Pharmacological treatments aim to reduce the effects of visual impairment. Restitutive interventions may include convergence training, pursuit training and saccade training. Compensative interventions may include training eye movements for reading, compensatory head posture or movements, use of eye blinks or colour cues and training in activities of daily living (Rowe 2011a). Substitutive interventions may include prisms, eye patches, lens alteration, extra ocular muscle surgery, botulinum toxin, magnification and environmental modification (Pigassou 1972). Pharmacological interventions may include prescription drugs such as baclofen, memantine and carbamazepine, and local anaesthetic injections (Choudhuri 2007; Thurtell 2010).

Commencement of interventions can be at multiple time points. People are offered interventions at different time points dependent on the time lapse since acquired brain injury onset, severity and type of symptoms and extent of recovery, if any. Some interventions can be used at any time point such as prisms, occlusion and lenses, whereas others - such as surgery - tend to be offered later once a visual condition is stable.

\section{How the intervention might work}

\section{Restitution}

Restitution includes the biochemical events that help restore functional neural tissue through the reduction of oedema, absorption of blood, restoration of normal neuronal physiology, and restoration of axon transport (Pollock 2011). Treatments of positive fusional amplitudes and stereopsis through repetition training of specific deficient functions, such as convergence insufficiency, have been reported as effective (Kerkhoff 2000). Restitutive interventions will include those where there is direct training of the impaired function or repetitive stimulation of eye movement.

\section{Compensation}

Compensation aims to improve the mismatch between the participants' skills and the demands placed on them by their environment, by teaching participants to compensate or adapt using a spared or intact function (Kerkhoff 1999; Kerkhoff 2000).

\section{Substitution}

Substitution involves adaptation of visual components that have been lost or disrupted through the use of optic devices, extra-ocular muscle surgery, botulinum toxin or environmental modifications (Kerkhoff 1999; Kerkhoff 2000). 


\section{Pharmacology}

Pharmacology aims to improve visual functioning through alteration of biochemical and/or physiological effects in the body. Gabapentin is proposed to interact with a high affinity binding site in brain membranes linked to calcium channels. It increases GABA synthesis. Baclofen is a derivative of GABA. It is proposed to activate GABA receptors and acts as an inhibitory neurotransmitter by blocking monosynaptic and polysynaptic reflexes. Aminopyridines are proposed to bind to open and non-conducting aspects of potassium ion channels resulting in prolonged action potentials and allowing increased neurotransmitter release at the neuromuscular junction.

\section{Why it is important to do this review}

There are many forms of interventions for eye movement disorders that occur following acquired brain injury. Although the natural history of acquired brain injury differs dependent on the cause, the treatment of the resultant eye movement disorder is principally the same regardless of cause. Although the cause may recover or progress, the resultant eye movement disorder may persist and requires treatment to alleviate or reduce visual symptoms. We have considered all interventions for all types of eye movement disorders regardless of cause and evolution of eye movement disorder. We extracted data for improving, stable or deteriorating eye movement disorders.

There are multiple time points at which interventions for eye movement disorders that occur following acquired brain injury may be provided. The timing of provision of treatment for eye movement disorders differs for the various treatment options. In the early stages, treatment may include conservative options such as eye patching or prisms which are used whilst the eye movement disorder is monitored until it improves or stabilises. Where eye movement disorders persist, further treatment options such as botulinum toxin or extra ocular muscle surgery may then be considered. There can be delays in referral of participants with acquired brain injury to eye care services and thus participants may not receive treatment early even though treatment options are available. This delay may impede general rehabilitation.

A recent systematic review of interventions for eye movement disorders in stroke found insufficient evidence to reach conclusions about the effectiveness of those interventions for this group of participants (Pollock 2011). There was an absence of relevant evidence and the authors gave an urgent recommendation for high-quality research. Furthermore, there is no consensus for what constitutes the optimum timing for commencing interventions for eye movement disorders due to stroke. Timing may vary dependent on the type of treatment being considered (e.g. eye patch versus extra-ocular muscle surgery) and/or the extent of visual symptoms (minimal versus severe impact on daily life). Because this review only concentrated on stroke populations, one recommendation was for a systematic review of interventions for eye movement disorders in participants with acquired brain injury in order to synthesise the current evidence base, to guide current practice and aid in the development of well-designed randomised controlled trials (RCTs). Other publications reporting efficacy and timing of interventions for eye movement disorders include populations with varied causes of brain damage such as tumours, inflammation, infection, stroke and metabolic causes.

\section{Purpose}

We have undertaken a high-quality systematic review of the existing evidence base in order to determine the evidence for effectiveness and timing of any treatment or management approaches for all adult participants with acquired brain injury with eye movement disorders. This review directly addressed the recommendations of the Cochrane systematic review on interventions for eye movement disorders in stroke (Pollock 2011), i.e. conduct a systematic review of interventions for eye movement disorders in participants with acquired brain injury in order to synthesise the current evidence base. This review examined the timing of interventions for eye movement disorders. The impact on objective and subjective measures of ocular alignment and motility are reported. This review can be used to guide current practice and aid in the development of well-designed RCTs which follow the UK Medical Research Council guidance on developing and evaluating complex interventions (Craig 2008).

\section{O B JECTIVES}

We aimed to assess the effectiveness of any intervention and determine the effect of timing of intervention in the treatment of strabismus, gaze deficits and nystagmus due to acquired brain injury. We considered restitutive, substitutive, compensatory or pharmacological interventions separately and compared them to control, placebo, alternative treatment or no treatment for improving ocular alignment or motility (or both).

\section{METHODS}

\section{Criteria for considering studies for this review}

\section{Types of studies}

We included randomised controlled trials (RCTs) and quasi-RCTs. We included cross-over trials if sufficient time was justified for the wash-out period between interventions to avoid carry-over effects, or where the condition was stable prior to recruitment, and where long-term follow-up was not required.

\section{Types of participants}

We included individuals with eye movement disorders due to acquired brain injury. We included trials of mixed aetiologies where more than $50 \%$ of participants had acquired brain injury. We excluded participants with primary diagnoses of multiple sclerosis (an immune-modulated process affecting the central nervous system, causing damage to the myelin sheath of nerve fibres) and degenerative conditions of the brain (such as Parkinson's disease, Huntingdon's disease and Alzheimer's). The type of eye movement disorder could include III, IV, and VI cranial nerve palsy, reduced fixation, gaze holding, horizontal or vertical gaze palsy (or both), internuclear ophthalmoplegia, one and a half syndrome, saccadic problems, smooth pursuit problems, strabismus, nystagmus, reduced convergence or divergence, conjugate deviation and skew deviation. The deviation of eye movement could be horizontal, vertical or torsional and the severity of eye movement disorder could be slight, small, moderate or marked, and could include paralysis or paresis. We included monocular and binocular eye movement disorders.

We accepted studies that included participants based on symptoms which can be assumed to be present as a direct result of an eye movement disorder. Symptoms could include double vision, 
blurred vision, reading difficulty, wobbling or jumbled vision and excessive head movements. We considered participants of all ages from studies of adults and children.

\section{Types of interventions}

We included any intervention that aimed to improve the defects of eye movement, or alleviate or reduce the visual symptoms associated with the disorder. We classified interventions as restitution, substitution, compensation or pharmacological. We included trials that documented the timing (recorded as time period from the onset of eye movement disorder) of any intervention that aimed to improve the defects of eye movement, or alleviate or reduce the visual symptoms associated with the disorder. We classified timing as early or late intervention, where early constituted intervention within one month of eye movement disorder onset.

\section{Types of outcome measures}

Where possible, we assessed each outcome as a dichotomous variable (yes or no) at the end of the intervention period and at a follow-up point (ideally a minimum of three months after the completion of the intervention and a maximum of 12 months after).

\section{Primary outcomes}

- Improvement in ocular motility measured by orthoptic assessments of reduction in the angle of deviation (within 10 prism dioptres of ortho/straight position) and/or extent of eye movement range (improvement of one or more grades of limitation ranging from 1 to 4), such that visual axes are aligned in primary or secondary gaze positions (or both)

\section{Secondary outcomes}

- Achievement of binocular single vision as assessed by cover test, motor fusional vergences and stereoacuity

- Reduction of, or alleviation of, participant-reported symptoms assessed by participant record notes or questionnaire

- Improvement in functional ability measured by validated measures such as activity of daily living questionnaires

- Quality of life data - any measure of participant or parent satisfaction relating to improvement in appearance or improvement to lifestyle

\section{Adverse events}

- Intractable permanent diplopia, perforating injury, symptomatic over- or under-correction, death

- Assessed by descriptive documentation

\section{Search methods for identification of studies}

\section{Electronic searches}

The Cochrane Eyes and Vision Information Specialist conducted systematic searches in the following databases for randomised controlled trials and controlled clinical trials. There were no language or publication year restrictions. The date of the search was 26 June 2017.

- Cochrane Central Register of Controlled Trials (CENTRAL; 2017, Issue 5) (which contains the Cochrane Eyes and Vision Trials Register) in the Cochrane Library (searched 26 June 2017) (Appendix 1);
- MEDLINE Ovid (1946 to 26 June 2017) (Appendix 2);

- Embase Ovid (1980 to 26 June 2017) (Appendix 3);

- CINAHL (Cumulative Index to Nursing and Allied Health Literature) (1982 to 26 June 2017) (Appendix 4);

- AMED (Allied and Complementary Medicine Database) (1985 to 26 June 2017) (Appendix 5);

- PsycINFO (1967 to 26 June 2017) (Appendix 6);

- Dissertations \& Theses (PQDT) database (http:// pqdtopen.proquest.com/search.html; searched 26 June 2017) (Appendix 7);

- PsycBITE (Psychological Database for Brain Impairment Treatment Efficacy) (http://www.psycbite.com/search.php: searched 26 June 2017) (Appendix 8);

- ISRCTN registry (www.isrctn.com/editAdvancedSearch; searched 26 June 2017) (Appendix 9);

- US National Institutes of Health Ongoing Trials Register ClinicalTrials.gov (www.clinicaltrials.gov; searched 26 June 2017) (Appendix 10);

- Health Services Research Projects in Progress (HSRProj) (wwwcf.nlm.nih.gov/hsr_project/home_proj.cfm; searched 26 June 2017) (Appendix 11);

- National Eye Institute Clinical Studies Database (clinicalstudies.info.nih.gov/cgi/protinstitute.cgi?NEl.0.html; searched 26 June 2017) (Appendix 12);

- World Health Organization International Clinical Trials Registry Platform (www.who.int/ictrp; searched 26 June 2017) (Appendix 13).

\section{Searching other resources}

We searched the reference lists of included trials to identify any further studies and checked the references of review articles about vision after acquired brain injury. We performed citation tracking using Web of Science Cited Reference Search for all included studies and contacted experts in the field, including authors of included trials and excluded studies identified as possible preliminary or pilot work. We searched reference material supplied by commercial companies who provide interventions aimed at restoration of eye movements.

We handsearched the following resources from their inception to the current date at http://pcwww.liv.ac.uk/ ${ }^{\sim}$ rowef/index_files/ Page646.htm.

- British and Irish Orthoptic Journal

- Australian Orthoptic Journal

- Proceedings of the European Strabismological Association (ESA)

- International Strabismological Association (ISA)

- International Orthoptic Association (IOA)

\section{Data collection and analysis}

We followed guidance in Chapter 7 of the Cochrane Handbook for Systematic Reviews of Interventions for data collection and Chapter 9 of the Cochrane Handbook for Systematic Reviews of Interventions for data analysis (Deeks 2011; Higgins 2011a). We also conformed to the Preferred Reporting Items for Systematic Reviews and MetaAnalyses (PRISMA) checklist (Moher 2009). The Cochrane Eyes and Vision group Information Specialist ran all the electronic searches, downloaded references into bibliographic software, and removed duplicates. Two review authors excluded any titles or abstracts 
which were obviously not related to acquired brain injury and vision. They independently considered each of these titles and abstracts and excluded any studies where the intervention was not specifically aimed at improving the eye movement disorder or the participant's ability to cope with the eye movement disorder. We resolved any disagreements through discussion. We obtained the full papers for any studies included at this stage.

\section{Selection of studies}

Two review authors independently applied the selection criteria, considering and documenting the type of studies, type of participants, intervention, comparison intervention, and the outcome measures. Each review author classified each study as 'include' or 'exclude'. For disagreement between these two review authors, consensus was planned through discussion involving a third review author. In practice, this was not required.

We listed all excluded studies (that we had obtained a full-text copy for) that included participants with eye movement disorders in the Characteristics of excluded studies table with the reasons for exclusion. We did not list studies in the Characteristics of excluded studies table that were excluded because they included participants that did not have eye movement disorders (i.e. visual neglect, age-related visual problems, or visual field loss), unless the two review authors agreed that there was a clear reason to do so.

\section{Data extraction and management}

We used a pre-designed data extraction form to record data from the included studies. Two review authors independently documented information found in the table in Appendix 14. If there were any discrepancies between data extracted by the two review authors, they were resolved through discussion. Data were entered into Review Manager 5 (Review Manager 2014).

\section{Assessment of risk of bias in included studies}

We used Cochrane's tool for assessing risk of bias for randomised trials as outlined in Chapter 8 of the Cochrane Handbook for Systematic Reviews of Interventions (Higgins 2011b). We assessed sequence generation, allocation concealment, masking (blinding) of participants, personnel and outcome assessors, incomplete outcome data and selective outcome reporting. We judged each domain as "low risk of bias", "high risk of bias" or "unclear". We also considered additional potential sources of bias for cross-over studies (Higgins 2011c), namely: (1) whether the cross-over design is suitable; (2) whether there is a carry-over effect; (3) whether only first period data are available; (4) incorrect analysis; and (5) comparability of results with those from parallel-group trials.

\section{Measures of treatment effect}

There was only one study that reported outcomes in a way that could be analysed in this review. This parallel group study reported dichotomous outcomes (reduction in angle of deviation within 10 prism dioptres, achievement of binocular single vision). We analysed these as risk ratios with $95 \%$ confidence intervals.

Most (four out of five) studies were cross-over trials. Ideally we would have extracted the results of paired analyses, but these were not available.

See Differences between protocol and review for further discussion of our planned methods.

\section{Unit of analysis issues}

The interventions are normally applied at individual level. Some interventions (e.g. prisms for diplopia or field defect, monocular occlusion) are used to treat only one eye. In these instances, there is no unit of analysis issue as the unit of randomisation is the same as the unit of analysis. However, some interventions (e.g. scanning eye exercises, extra-ocular muscle surgery, pharmacological interventions) treat both eyes. We report outcome measures for the person.

We followed guidance in the Cochrane Handbook for Systematic Reviews of Interventions for the incorporation of data from crossover trials, i.e. estimating mean and standard errors from paired analysis (Higgins 2011c).

\section{Dealing with missing data}

If an included study did not report a particular outcome, we did not include that study in the analyses of that outcome. If an included study had missing data (e.g. participants lost to follow-up) we reported results for included participants based on the raw data provided. We used all trial data even if intention-to-treat analysis was not conducted. However, we looked at which participants dropped out or changed treatment to determine if these related to one specific group or were dispersed across all groups. We also contacted trial authors for missing data.

\section{Assessment of heterogeneity}

We assessed for heterogeneity primarily by examining the characteristics of the study. We did not conduct a meta-analysis due to clinical heterogeneity, so we did not implement our other planned methods for assessing heterogeneity. See Differences between protocol and review.

\section{Assessment of reporting biases}

We assessed the possibility of selective outcome reporting as detailed in the Assessment of risk of bias in included studies section. We did not conduct any meta-analyses so we did not use our planned methods for assessing publication bias. See Differences between protocol and review.

\section{Data synthesis}

We provide a narrative account of the data available. See Differences between protocol and review for details of planned methods for data synthesis that were not used.

\section{Subgroup and sensitivity analyses}

Due to the small number of studies identified, we were unable to conduct our planned subgroup and sensitivity analyses. See Differences between protocol and review.

\section{'Summary of findings' table}

We aimed to prepare a 'Summary of findings' table for each relevant comparison including all pre-specified outcomes:

- improvement in ocular motility;

- achievement of binocular single vision;

- improvement in functional ability;

- quality of life;

- adverse effects. 
The cross-over studies were not reported in a way that permitted calculation of effect sizes so we have reported the direction, rather than the size of the effect in these cases. We graded the certainty of the evidence using the GRADE approach (Guyatt 2008; GRADEpro 2014). In the absence of pooled data we were guided by the implementation of GRADE for a narrative summary (Murad 2017). We considered methodological limitations of the included studies, indirectness, imprecision, inconsistency and likelihood of publication bias. We did not pre-specify the 'Summary of findings' table and GRADE assessment in the protocol. See Differences between protocol and review.

\section{RES U LTS}

\section{Description of studies}

\section{Results of the search}

The electronic searches yielded a total of 5049 references (Figure 1). The Cochrane Information Specialist removed 330 duplicate records and we screened the remaining 4719 reports. We rejected 4700 records after reading the abstracts and obtained the fulltext reports of 19 references for further assessment. We included five reports of five studies (Averbuch-Heller 1997; Kalla 2011; Lee 1994; Strupp 2003; Thiagarajan 2014) and we excluded 14 studies (see Characteristics of excluded studies for details). We did not identify any ongoing studies from our searches of the clinical trials registries. 
Figure 1. Study flow diagram

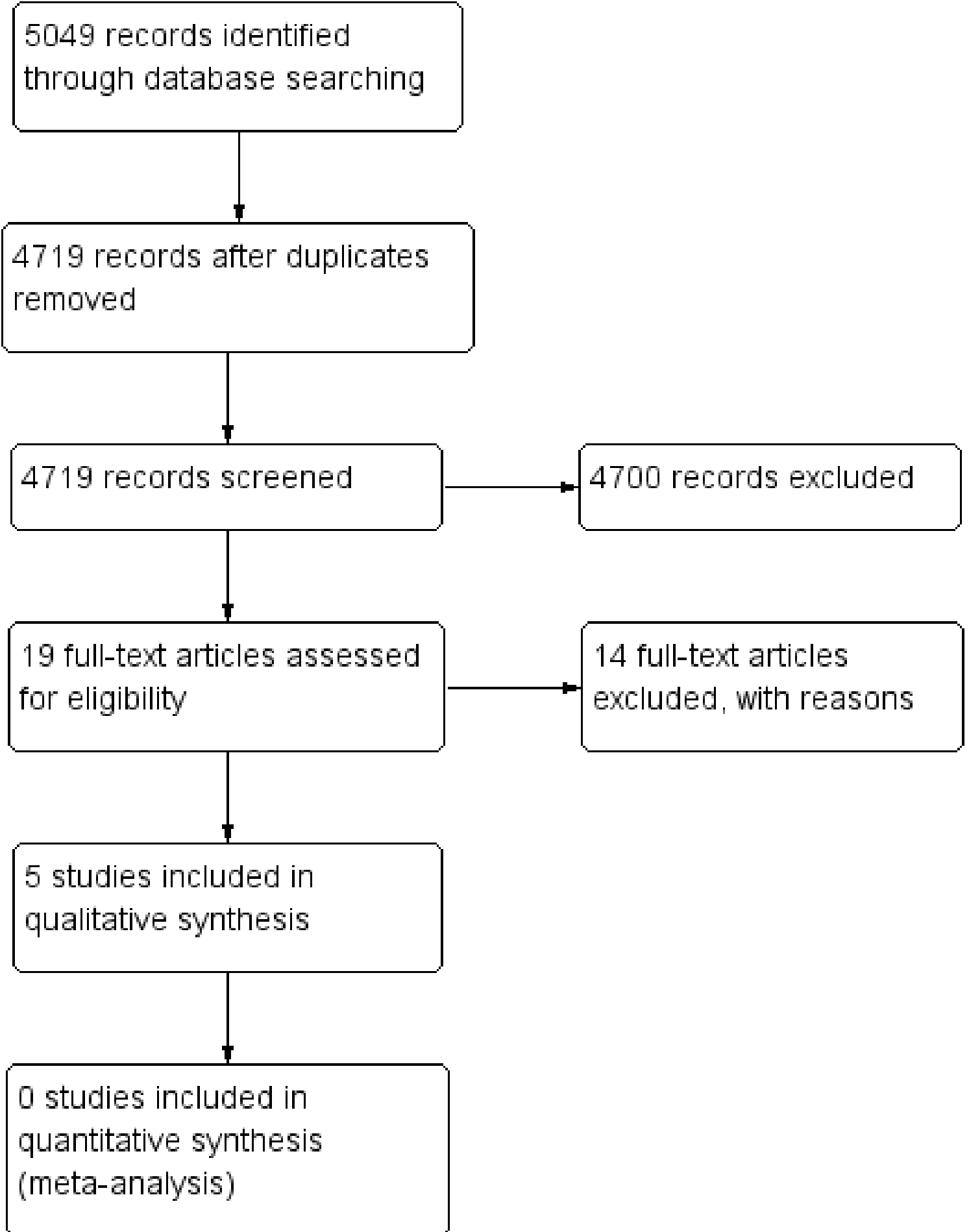

\section{Included studies}

We included five trials which are summarised below. Additional details can be found in the Characteristics of included studies tables.

Averbuch-Heller 1997 randomised 21 participants (10 females and 11 males) aged 25 to 73 years. There were 15 cases of acquired pendular nystagmus and six of jerk nystagmus. The trial was conducted across four sites in the USA and Germany. It was a double-masked randomised controlled trial with a cross-over design, which compared two pharmacological interventions: 10 $\mathrm{mg}$ baclofen and $30 \mathrm{mg}$ gabapentin. Drug 1 was taken for two weeks, followed by a wash-out period of two weeks; and drug 2 was then taken for two weeks (six-week study duration in total). The participant group was considered homogenous in that they had both interventions. There were no exclusions, but one participant 
was lost to follow-up. Outcomes were measured at baseline and at two-, four- and six-week follow-up periods. They included measurement of Landolt C visual acuity, eye movement recordings, subjective image motion, adverse drug effect reporting and choice of continued treatment. Inclusion criteria were specified, but not exclusion criteria.

Kalla 2011 randomised eight participants (six females and two males) with acquired downbeat nystagmus, aged 58 to 76 years. The trial was conducted at one site in Germany. It was a double-masked randomised controlled trial with a cross-over design, which compared two pharmacological interventions: 4aminopyridine at a $10 \mathrm{mg}$ dose and 3,4-diaminopyridine at a 10 $\mathrm{mg}$ dose. Drug 1 was taken for one day, followed by a wash-out period of six days; and Drug 2 was taken for one day (eight-day study duration in total). The participant group was considered homogenous in that they had both interventions. There were no exclusions or losses to follow-up. Outcomes included measurement of $3 \mathrm{D}$ video-oculography and participant recall of medical side effects. Inclusion criteria were specified, but not exclusion criteria.

Lee 1994 recruited and randomised 54 people presenting with acute unilateral sixth nerve palsy. Forty-seven participants were followed up. There two groups: those receiving botulinum toxin (Dysport $^{\mathrm{TM}}$ ) to the isolateral medial rectus muscle (22 participants), and those observed for recovery with no invasive treatment $(25$ participants). This trial was conducted at one site in the UK. The intervention arm comprised 13 males and 9 females, with a mean age of 63 years (range: 24 to 83). The control arm comprised 12 males and 13 females with a mean age of 61 years (range 24 to 86). This was a parallel design randomised controlled trial. Outcome measurements included range of ocular movements for abduction defect, angle of deviation measured by prism cover test, and field of binocular single vision. These groups were compared to each other for clinical diagnosis of recovery. A full recovery was defined as completely normal ocular rotations with full field of binocular single vision. Stable recovery was defined as normal binocular single vision with a minor asymptomatic abduction defect or a small asymptomatic vertical deviation. Non-recovery was defined as a persisting esotropia in primary position with diplopia not controllable by normal amplitudes of fusional vergence. Two control participants were excluded due to change of diagnosis and four were lost to follow-up. One participant from the botulinum toxin group was lost to follow-up. Follow-up ranged from 4 to 42 months. Both groups were considered homogenous as gender, age range, aetiology of sixth nerve palsy, duration of symptoms and laterality of palsy were similar across both groups. The mean deviation of control participants was 17.8 prism dioptres (PD); for botulinum toxin participants this was 28.6 PD. The difference in deviation across both groups was significant $(P=0.02)$. Three of the 22 participants who received botulinum toxin injection had one repeat injection.
Strupp 2003 recruited 18 participants with acquired downbeat nystagmus (nine females and nine males) aged 50 to 85 years. One person was subsequently excluded due to alcohol misuse. The trial was conducted at one site in Germany. This was a doublemasked randomised controlled trial with a cross-over design, which compared one pharmacological intervention with placebo (3,4-Diaminopyridine at $20 \mathrm{mg}$ dose versus lactose). Drug 1 was taken for one day, followed by a wash-out period of more than one week; and Drug 2 was taken for one day (nine-day study duration in total). The participant group was considered homogenous in that they had both interventions. There was one exclusion (due to alcohol abuse), but no loss to follow-up. Outcomes included measurement at baseline and follow-up of 2D video-oculography and participant reports of oscillopsia and recall of medical side effects. Inclusion and exclusion criteria were specified.

Thiagarajan 2014 randomised 12 participants with mild traumatic brain injury which happened more than one year previously. Participants aged on average $29 \pm 3$ years. The trial was conducted at one site in the USA. It was a single-masked randomised controlled trial with a cross-over design, which compared oculomotor rehabilitation versus sham treatment. Oculomotor treatment involved two sessions per week, each session lasting 60 minutes. Participants were provided with training of version and vergence eye movements and accommodation for 15 minutes each, interspersed with five-minute rest periods. Sham treatment involved two sessions of basic reading tasks per week, each session lasting 60 minutes. Each treatment was delivered for a period of six weeks, with a one-week washout period between treatments and after the second treatment. The participant group was considered homogenous in that they had both interventions. There were no exclusions or losses to follow-up. Outcomes were measured at baseline, after the first treatment block and after the second treatment block. They included infrared eye recording of reading eye movements, measurement of reading rate, saccadic ratio, near point of convergence, binocular accommodative amplitude and use of the convergence insufficiency symptom scale questionnaire. Inclusion and exclusion criteria were specified.

\section{Excluded studies}

We excluded 14 studies (Barton 1994; Cifu 2014; Claassen 2013; Clement 2007; Dai 2003; Feil 2013; Gur 1992; Leigh 1991; Leivo 1996; Lorenz 2006; Metz 1988; Sharpe 2005; Strupp 2008; Zampieri 2009). For reasons of exclusion, please see the Characteristics of excluded studies table. Main reasons for exclusion were that the studies included conditions that were listed in our exclusion criteria such as multiple sclerosis and degenerative conditions, or that studies were not RCTs.

\section{Risk of bias in included studies}

See Figure 2; Figure 3. 
Figure 2. 'Risk of bias' graph: review authors' judgements about each 'Risk of bias' item presented as percentages across all included studies.

Random sequence generation (selection bias)

Allocation concealment (selection bias)

Blinding of participants and personnel (performance bias)

Blinding of outcome assessment (detection bias)

Incomplete outcome data (attrition bias)

Selective reporting (reporting bias)

Other bias

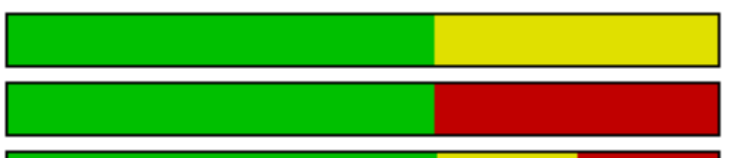

L

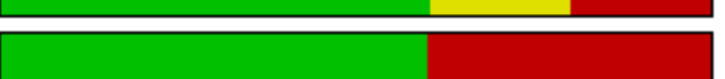

$$
\text { L }
$$
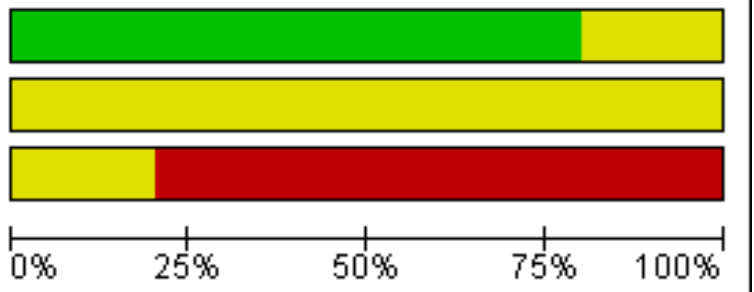

Low risk of bias

Unclear risk of bias

High risk of bias 
Figure 3. 'Risk of bias' summary: review authors' judgements about each 'Risk of bias' item for each included study.

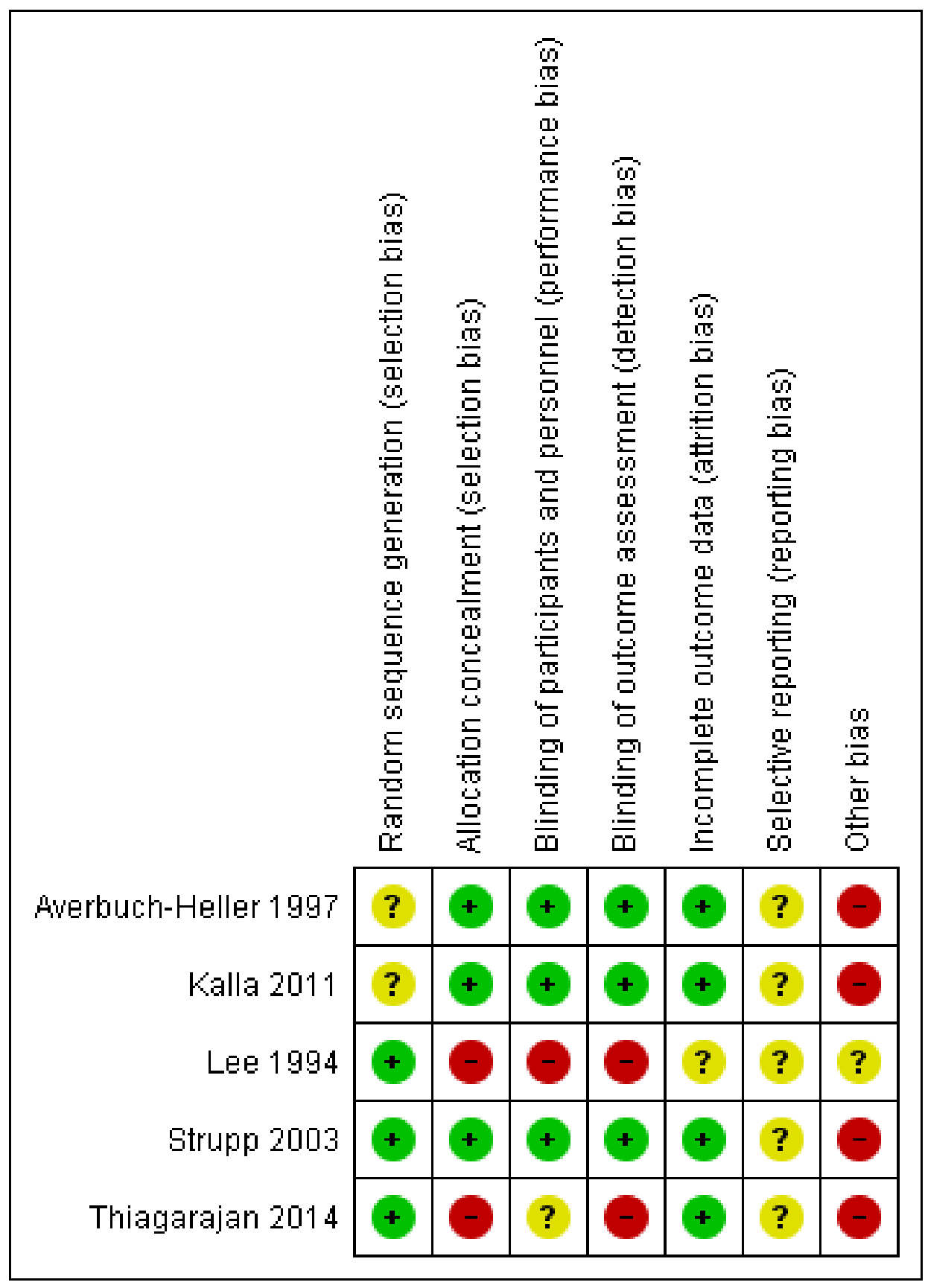

\section{Allocation}

Sequence generation was unclear in two trials (Averbuch-Heller 1997; Kalla 2011). It was evident that randomisation had occurred but the method was not stated. One study (Lee 1994) specified the use of a random number table; another (Strupp 2003) used a computer-generated randomisation list; and a third (Thiagarajan 2014) used odd/even number randomisation for sequence generation. We considered these three trials to have low risk of bias for this domain.

Adequate prevention of knowledge of the allocated interventions was achieved in three trials (Averbuch-Heller 1997; Kalla 2011;
Strupp 2003). These trials used double-masked designs, with investigators and participants masked to the allocation of interventions. We judged that allocation concealment did not take place in the other two studies (Lee 1994; Thiagarajan 2014).

\section{Blinding}

Masking of participants and personnel:

Adequate prevention of knowledge of the allocated interventions was achieved in three trials (Averbuch-Heller 1997; Kalla 2011; Strupp 2003). These trials used double-masked designs, with investigators and participants masked to the allocation of 
interventions. There was no masking (blinding) of investigators or participants in the other two trials (Lee 1994; Thiagarajan 2014). We judged Lee 1994 to be at high risk of both performance and detection bias. Thiagarajan 2014 was single-masked (participants were masked to the intervention) but all testing and training was delivered by one investigator. We were less sure as to the extent to which this lack of masking would create performance bias that was independent of the effect of the intervention.

Masking of outcome assessors:

Adequate prevention of knowledge of the allocated interventions was achieved in three trials (Averbuch-Heller 1997; Kalla 2011; Strupp 2003) in which the outcome assessors were masked to the allocation of interventions. There was no masking of outcome assessors for the remaining studies (Lee 1994; Thiagarajan 2014).

\section{Incomplete outcome data}

Incomplete outcome data was adequately addressed in all four trials, for which a low risk of bias was determined. All participants were accounted for throughout each trial, with outcome data provided for participants completing the trials and information provided for any participants lost to follow-up or excluded. One study (Averbuch-Heller 1997) reported one person lost to followup and this participant was removed from analysis, with followup equal across groups. One study (Strupp 2003) excluded one participant and removed the related results from analysis, with follow-up equal across groups. In Lee 1994, follow-up was clearly described and most participants were followed up (47/54, 87\%). However, loss to follow-up appeared to occur predominantly in the control group. It is unclear whether this could have impacted a comparison between control and intervention groups.

\section{Selective reporting}

It was difficult to reliably judge the extent of selective reporting due to lack of published protocols and the fact the trials were unregistered. Complicated analyses may also have been subject to selective reporting.

\section{Other potential sources of bias}

We considered the following additional sources of bias for crossover studies.

\section{Was the cross-over design suitable?}

We judged the trials of pharmacological interventions possibly suitable for cross-over design but the oculomotor rehabilitation study (Thiagarajan 2014) we were less sure that this was suitable as the training received at the first phase may influence the second phase.

\section{Was there a carry-over effect?}

Possibly not in the drug trials where there was a washout phase but this was unclear because none of the studies addressed this issue directly.

\section{Was only first period data are available?}

Data were not reported fully and so neither first nor second period data were available.

\section{Was the analysis correct?}

This was difficult to judge. None of the studies reported appropriate effect estimates and standard error which would have permitted analysis in this review. One study reported findings for the intervention group only (Thiagarajan 2014).

\section{Comparability of results with those from parallel-group trials}

There was no overlap in cross-over and parallel-group trials.

\section{Effects of interventions}

See: Summary of findings for the main comparison Botulinum toxin versus observation; Summary of findings 2 Pharmacological treatment

Given the differences between studies, it was not possible to provide a meta analysis of results. Each study is discussed individually.

\section{Restitutive interventions}

We did not identify any relevant studies of restitutive interventions.

\section{Substitutive interventions}

We identified one trial of botulinum toxin compared with observation for acute sixth nerve palsy (Lee 1994); see Summary of findings for the main comparison.

\section{Improvement in ocular motlity / Achievement of binocular single vision}

At four months after entry into the trial, Lee 1994 reported full recovery with reduction in angle of deviation within 10 prism dioptres in 21/22 (95\%) participants given botulinum toxin and $20 / 25(80 \%)$ of control participants (risk ratio $1.19,95 \% \mathrm{Cl} 0.96$ to 1.48; number of participants $(n)=47$; Table 1 ). These same participants also achieved binocular single vision. We judged the certainty of evidence as low, downgrading for risk of bias and imprecision. It was not possible to mask investigators or participants to allocation and there was variable follow-up between groups.

\section{Adverse effects}

In the injection group only, there were cases of transient ptosis in $2 / 22(9 \%)$ of participants, and transient vertical deviation in $4 / 22$ (18\%). In total the risk of complications per injection was $24 \%$ and the risk of complications per participant was $27 \%$.

\section{Quality of life / Improvement in functional ability}

The study did not report on functional ability and quality of life.

\section{Compensatory interventions}

We identified one cross-over trial of a compensatory intervention (Thiagarajan 2014). This trial compared oculomotor rehabilitation with sham training in 12 people with mild traumatic brain injury (Table 2). We have some doubts as to whether a cross-over study design is appropriate since this is an intervention that may have a permanent effect, i.e. there may be an interaction between phase and effect. The data were not analysed in a way that permitted analysis of phase 1 only. The cross-over study design appeared to be ignored in the analysis. 


\section{Improvement in ocular motility}

With these caveats in mind, the study reported a drop in mean saccade ratio with oculomotor rehabilitation from a mean value of 2.1 to 1.7. This change was reported to be statistically significant ( $P$ $<0.05)$. The saccade ratio was defined as the number of tracking saccades executed divided by the number of test target step displacements, with a ratio of 1 being optimal. The saccade ratio was tested on single and multiple lines. The drop in reading single lines was from 2.7 to 2.2 but this change reported as not statistically significant (no $P$ value reported).

\section{Improvement in functional ability}

Reading rate (words per minute) increased with oculomotor rehabilitation from 142 (standard deviation (SD) 10) to 177 (SD 14) $(P<0.01)$. Reading levels increased by two grades, from 4.1 (SD 0.7) before oculomotor training to 6.3 (SD1.2) after training. There was also a reduced number of fixations per 100 words, from 164 (SD 10) to 135 (SD 11). Binocular accommodative amplitude and near point of convergence improved, alongside a reduction in near fixation symptoms.

Data from the sham training group were not reported but it was stated that the change in parameters was non-significant.

\section{Achievement of binocular single vision / Quality of life / Adverse effects}

This study did not report on binocular single vision, quality of life or adverse effects.

\section{Summary}

We considered this evidence to be very low-certainty. We downgraded by two levels because of the study limitations described above, in particular the limitations of a cross-over study design for this intervention, and the lack of data reported from the control (no intervention) group. We downgraded by one level for imprecision due to the small number of participants.

\section{Pharmacological interventions}

See Summary of findings 2.

We identified three cross-over studies of pharmacological interventions (Averbuch-Heller 1997; Kalla 2011; Strupp 2003).

These studies investigated two classes of pharmacological interventions: GABAergic drugs (gabapentin; baclofen) and aminopyridines (4-aminopyridines (AP); 3,4-diaminopyridine (DAP)).

One study compared gabapentin and baclofen (Averbuch-Heller 1997, $n=21$ ); one study compared 3,4-DAP with placebo (Strupp $2003, n=17$ ); and one study compared 4-AP with 3,4-DAP (Kalla $2011, n=8)$.

The results are summarised in Table 3.

\section{Gabapentin versus baclofen}

\section{Improvement in ocular motility}

Averbuch-Heller 1997 included 21 participants but reported results separately for acquired pendular nystagmus $(n=15)$ and jerk nystagmus $(n=6)$. It was difficult to extract data on comparative effectiveness from the study report, as the data were only reported in scatter plots separately by intervention and comparator. We have to rely on the authors' statements in the text which were as follows:

\section{Pendular nystagmus}

- "...median eye speed was reduced in all three planes by gabapentin, during viewing of the near or far targets; the effects were similar in darkness. The predominant frequency of oscillation was reduced, on average, by less than $9 \%$ by gabapentin, but this reduction was consistent and statistically significant $(P<0.05)$ in the horizontal and vertical planes during viewing of far and near visual targets."

- "...median eye speed was reduced significantly $(P<0.005)$ only in the vertical plane by baclofen. Baclofen produced no significant changes in the predominant frequency of APN."

- "Neither drug caused any significant change in the gain of saccades, smooth pursuit or the VOR [vestibulo-ocular reflex], or changes in saccadic velocity, or conjugacy, similar to reported effects in normal subjects. This was the case even in those patients who showed substantial reduction of nystagmus with gabapentin."

\section{Jerk nystagmus}

- "For this group of patients, neither drug produced a significant change in median, slow-phase eye speed. Changes in individual patients depended on whether they viewed the near or far targets."

As there were fewer participants in this group, it is difficult to know whether the lack of statistical significance was attributable to a different effect in this subgroup or to the different number of participants. Results for individual participants were reported but these are difficult to interpret.

\section{Achievement of binocular single vision}

This was not reported but the results of the visual acuity tests were as follows.

\section{Pendular nystagmus}

- "...there was a significant $(P<0.006)$ improvement of visual acuity with gabapentin; this improvement was greater during testing with the near card."

- "...baclofen produced no significant change in visual acuity measured at near or at far."

\section{Jerk nystagmus}

- "... neither drug produced a significant change in visual acuity."

\section{Reduction in participant-reported symptoms}

Results for oscillopsia were as follows.

\section{Pendular nystagmus}

- "Twelve patients reported some illusory motion of the visual target before treatment, and gabapentin reduced this oscillopsia in 6."

- "...Baclofen changed the direction of oscillopsia, without reducing it, in 2 patients." 


\section{Jerk nystagmus}

- "Four patients reported some illusory motion of the visual target before treatment; neither drug influenced oscillopsia in 4."

\section{Quality of life}

This outcome was not reported but ten participants with pendular nystagmus chose to continue treatment with gabapentin and one chose to continue treatment with baclofen. Two participants with jerk nystagmus chose to continue treatment with gabapentin and one chose to continue with baclofen.

\section{Adverse effects}

No significant adverse events were reported. Drug intolerance was reported in one person in the gabapentin group and four participants in the baclofen group. Adverse events of increased ataxia were reported in three participants in the gabapentin group and two participants in the baclofen group.

\section{Improvement in functional ability}

This outcome was not reported.

\section{Summary}

This study provides very low-certainty evidence that gabapentin is better than baclofen and that these effects may be different in pendular and jerk nystagmus. The data reported by this study did not allow us to estimate the size of the effect. The lack of a formal subgroup analysis means that it is unclear if the difference between the two types of nystagmus may be a chance finding. The evidence is low-certainty because of the small numbers of participants (for which we downgraded by two levels for imprecision) and risk of bias (it was a cross-over study with analysis that did not permit estimation of effect size).

\section{3,4-diaminopyridine versus placebo}

One study (Strupp 2003) compared 3,4-DAP (20 mg) with placebo (lactose) in 17 people with downbeat nystagmus. Assessments were made 30 minutes after taking the drug. Comparative measures of effect were not reported, and could not be calculated with the information given, but statistical tests accounted for the cross-over design (by two-way analysis of variance).

\section{Improvement in ocular motility}

In the 3,4-DAP group there was a reduction in mean peak slowphase velocity from 7.2 degrees/second (SD 4.2) to 3.1 degrees/ second (SD 2.5), which is considered clinically important. There was no change in the lactose group (7.4 degrees/second (SD 4.1) to 7.3 decrees/second (SD 3.7)). This difference between the groups was reported to be statistically significant $(P<0.001)$.

\section{Reduction in participant-reported symptoms}

The authors report that in 10 of the 17 participants, mean peak slow-phase velocity decreased by more than $50 \%$. These 10 people also reported having less oscillopsia.

\section{Adverse effects}

No significant adverse events were reported. Nine participants continued treatment. Three participants reported transient side effects of minor perioral/distal paraesthesia.

\section{Outcomes not reported}

- Achievement of binocular single vision

- Improvement in functional ability

- Quality of life

\section{Summary}

This study provides very low-certainty evidence that 3,4-DAP may reduce the mean peak slow-phase velocity in people with downbeat nystagmus. We judged the evidence to be very lowcertainty because of the small size of the study (for which we downgraded by two levels for imprecision) and risk of bias (it was a cross-over study with no comparative measure of effect reported).

\section{4-aminopyridines versus 3,4-diaminopyridine}

One study (Kalla 2011) compared 4-AP and 3,4-DAP (both given as a single $10 \mathrm{mg}$ dose) in eight people with downbeat nystagmus. Assessments were made 45 and 90 minutes after drug administration. Comparative measures of effect were not reported, and could not be calculated with the information given, but statistical tests probably accounted for the cross-over design ("repeated measurement analysis of variances").

\section{Improvement in ocular motility}

The authors report the following.

- Mean slow-phase velocity decreased from 5.68 degrees/second to: 3.29 degrees/second at 45 minutes, and 2.96 degrees/second at 90 minutes in people who had taken 3,4-DAP $(P<0.01)$.

- Mean slow-phase velocity decreased from 6.04 degrees/second to: 1.58 degrees/second at 45 minutes, and 1.21 degrees/second at 90 minutes in people who had taken 4-AP $(P<0.00001)$.

- The difference between the two drugs at 45 and 90 minutes was statistically significant $(P<0.05)$.

\section{Adverse effects}

"All 8 patients reported mild paraesthesias from 30 minutes to 2 hours after ingestion of both medications. No other side effects were reported."

\section{Outcomes not reported}

- Achievement of binocular single vision

- Reduction in participant-reported symptoms

- Improvement in functional ability

- Quality of life

\section{Summary}

This study provides low-certainty evidence that both 3,4-DAP and 4-AP may reduce the mean slow-phase velocity in people with downbeat nystagmus. This effect may be stronger with 4-AP. We judged the evidence to be very low-certainty because of the small size of the study (for which we downgraded by two levels for imprecision) and risk of bias (it was a cross-over study with analysis that did not permit estimation of effect size).

\section{Timing of interventions}

Three trials (Averbuch-Heller 1997; Kalla 2011; Strupp 2003) did not specify the timing at which interventions were provided after onset of the nystagmus. Therefore the timing of intervention cannot be 
classified as acute or long-term, and its effect in the treatment of nystagmus cannot be determined. In Lee 1994, participants were recruited at acute presentation to hospital emergency department. Effect of acute intervention can be considered for this trial (see section 2 above). In Thiagarajan 2014, participants were recruited at least one year post-incident to ensure that any subsequent changes during training were not secondary to any natural recovery period (estimated by the authors as being up to six to nine months postincident). Effect of late-timed intervention can be considered for this trial (see section 3 above).

\section{DISCUSSION}

\section{Summary of main results}

Following acquired brain injury, a variety of eye movement disorders may occur including: strabismus, ocular cranial nerve palsies, supranuclear and internuclear gaze palsies, nystagmus, saccadic and smooth pursuit palsies. The literature on interventions for these eye movement disorders consists predominantly of retrospective studies, cohort studies and case series, which are useful for describing various treatment options but do not allow the estimation of effect size for recommendations based on clinical and cost effectiveness.

We found five randomised controlled trials (RCTs), with a total of 116 participants, that were eligible for inclusion in this review. These trials included conditions of acquired nystagmus, sixth cranial nerve palsy and ocular motility defects induced by traumatic brain injury. We did not identify any relevant studies of restitutive interventions. In view of the differences between studies, it was not possible to provide a meta analysis of results.

We identified one trial of a substitutive intervention for acute sixth nerve palsy. At four months after entry into the trial, people given botulinum toxin were more likely to make a full recovery (with reduction in angle of deviation within 10 prism dioptres), compared with observation (low-certainty evidence). These same participants also achieved binocular single vision and had improved visual symptoms. In the injection group only, there were cases of transient ptosis in $2 / 22(9 \%)$ of participants, and transient vertical deviation in $4 / 22$ (18\%); and there was a total complication rate of $24 \%$ per injection and $27 \%$ per participant. Functional ability and quality of life were not reported. We judged the certainty of evidence as low, downgrading for risk of bias and imprecision.

We identified one cross-over trial of a compensatory intervention. This study took place in the USA. Oculomotor rehabilitation was compared with sham training in 12 people with mild traumatic brain injury at least one year post-incident. We judged the evidence from this study to be very low-certainty. The study was small, data for the sham training group were not fully reported, and it was unclear if a cross-over study design was appropriate as this is an intervention that may be expected to have a permanent effect.

We identified three cross-over studies of pharmacological interventions, which took place in Germany and the USA. These studies investigated two classes of pharmacological interventions: GABAergic drugs (gabapentin, baclofen) and aminopyridines (4aminopyridines (AP), 3,4-diaminopyridine (DAP)). We judged the evidence from all three studies as very low-certainty because of the small numbers of participants (for which we downgraded two levels for imprecision) and risk of bias (they were cross-over studies with data reported in a way that precluded estimation of effect size). One study compared gabapentin (up to $900 \mathrm{mg} /$ day) and baclofen (up to $30 \mathrm{mg} /$ day) in 21 people with pendular and jerk nystagmus and followed up to two weeks. This study provides very low-certainty evidence that gabapentin is better than baclofen and that these effects may be different in pendular and jerk nystagmus. There was no formal subgroup analysis, so it is unclear whether the difference between the two types of nystagmus was a chance finding or not. No significant adverse events were reported. Drug intolerance was reported in one person from the gabapentin group and four participants from the baclofen group. Increased ataxia were reported in three participants from the gabapentin group and two participants from the baclofen group. One study compared a single dose of 3,4-DAP (20mg) with placebo in 17 people with downbeat nystagmus. Assessments were made 30 minutes after taking the drug. This study provides very low-certainty evidence that 3,4-DAP may reduce the mean peak slow-phase velocity in people with downbeat nystagmus and this may be accompanied by less oscillopsia. Three participants reported transient side effects of minor perioral/distal paraesthesia. One study compared a single 10 $\mathrm{mg}$ dose of either 4-AP or 3,4-DAP, in eight people with downbeat nystagmus. Assessments were made 45 and 90 minutes after drug administration. This study provides very low-certainty evidence that both 3,4-DAP and 4-AP may reduce the mean slow-phase velocity in people with downbeat nystagmus. This effect may be stronger with 4-AP.

\section{Overall completeness and applicability of evidence}

Overall the evidence on interventions for eye movement disorders due to acquired brain injury is not complete. We identified only five relevant studies. These studies covered a heterogenous group of conditions and interventions. They had limitations in their design which means that it is difficult to draw firm conclusions as to the effects of the treatments studied. Furthermore, no information was obtained on the cost of treatment or on measure of participant or parent satisfaction relating to treatment options and effectiveness. It was, however, possible to describe the outcome of treatment in each trial and ascertain the occurrence of adverse events.

\section{Certainty of the evidence}

We judged the certainty of evidence for all outcomes to be very low or low primarily due to risk of bias in the included studies and imprecise effect estimates. Four of the five studies had a crossover design but relevant estimates of effect from an appropriate analyses were not reported in these studies. The included studies were small.

\section{Potential biases in the review process}

We included five trials in this review. There may be a risk of publication bias regarding other conducted, but not registered or published, trials. We systematically searched the available literature and did not identify further completed trials meeting our inclusion criteria.

We made some modifications to the protocol but none of these were likely to bias the results of the review. The protocol amendments are specified in Differences between protocol and review. The majority of the changes relate to methods that were not implemented because of a lack of data. The main qualitative change we implemented was to consider the different types of interventions separately. It is unlikely that this introduced bias, 
particularly as the data precluded formal meta-analysis. Other modifications included preparing a summary of findings table and GRADE assessment, as required by updated Cochrane methods. All outcomes pre-specified in the protocol were included in the summary of findings table.

\section{Agreements and disagreements with other studies or reviews}

Similar results to those in this systematic review were reported in another Cochrane systematic review on interventions for disorders of eye movement in participants with stroke (Pollock 2011). Their review included two trials of pharmacological treatment for nystagmus from which they concluded there was insufficient evidence to reach conclusions about the effectiveness of interventions for participants with eye movement disorders after stroke, and they recommended further high-quality research. Three of the five trials included in our systematic review were also trials of pharmacological treatments for nystagmus. We also found insufficient high-quality evidence for the treatment of eye movement disorders due to acquired brain injury.

There have been two recent reviews of the role of botulinum toxin in infantile esotropia (Issaho 2017) and strabismus (Mahan 2017). In both reviews only non-comparative and non-randomised comparative studies were included, which would not have been eligible for the current review. Both reviews concluded that the overall success rate of botulinum toxin in these groups of patients was $70 \%$ to $80 \%$.

It is important to acknowledge that certain interventions do not warrant randomised controlled trials to prove their effectiveness. For example, Fresnel prisms are an established intervention for correction of double vision (diplopia). These are temporary plastic press-on prisms that can be applied to spectacles and are used in selected cases of diplopia to correct the misalignment of the eyes that causes this symptom (Gunton 2012; Haller 2014). Although not a restorative treatment, these prisms alleviate the symptom of diplopia and are a clinical and cost-effective treatment option; and, likely, unethical to withhold as a treatment option. However, for persistent eye movement problems requiring longterm management, further high-quality randomised controlled trials are required of the various options for pharmacological and surgical treatment of eye movement disorders.

\section{AUTHORS' CONCLUSIONS}

\section{Implications for practice}

We were unable to fully address the aims and objectives of this review due to the limited number of trials identified, the low to very low-certainty evidence, and the variations in the conditions being treated. Gabapentin and 4-aminopyridine may reduce oscillatory eye movements and improve visual acuity and participant-reported symptoms. Botulinum toxin may improve participant symptoms earlier than observation in people with sixth nerve palsy. Very low-certainty evidence on oculomotor rehabilitation suggested improved reading ability, binocular accommodation aptitude and near point of convergence in comparison to sham training. Adverse events these were specific to the interventions but were mainly mild and transient.

\section{Implications for research}

There is a clear need for good quality trials to be conducted in order to improve the evidence base for restitutive, substitutive, compensatory and pharmacological therapies for eye movement disorders following acquired brain injury. Further research is also required to consider the effect of acute versus long-term timing of interventions for these conditions.

Standardisation of research design and core outcome measures is of importance taking into consideration the types and dosages of drugs. Key characteristics for a future trial would be: adequate sample size based on sound estimates of clinically meaningful effect size; multi-centre recruitment sites; computer-generated randomisation schedule with concealed allocation; use of a core outcome set and measures (COMET-initiative); a doublemasked trial design when possible (i.e. appropriate masking of participants and trial personnel); published protocol; and full outcome reporting following a pre-determined statistical analysis plan.

\section{ACKNOWLEDGEMENTS}

Cochrane Eyes and Vision created and executed the electronic search strategies. We thank Catey Bunce and Tracey Shipman for their comments. We thank Anupa Shah and Iris Gordon for their assistance throughout the review process. 


\section{R E F E R E N C E S}

\section{References to studies included in this review}

\section{Averbuch-Heller 1997 \{published data only\}}

Averbuch-Heller L, Tusa RJ, Fuhry L, Rottach KG, Ganser GL, Heide W, et al. A double-blind controlled study of Gabapentin and Baclofen as treatment for acquired nystagmus. Annals of Neurology 1997;41(6):818-25.

\section{Kalla 2011 \{published data only\}}

Kalla R, Spiegel R, Claassen J, Bardins S, Hahn A, Schneider E, et al. Comparison of 10-mg doses of 4-aminopyridine and 3,4diaminopyridine for the treatment of downbeat nystagmus. Journal of Neuro-Ophthalmology 2011;31(4):320-5.

\section{Lee 1994 \{published data only\}}

Lee J, Harris S, Cohen J, Cooper K, MacEwen C, Jones S. Results of a prospective randomised trial of botulinum toxin therapy in acute unilateral sixth nerve palsy. Journal of Pediatric Ophthalmology and Strabismus 1994;31(5):283-6.

\section{Strupp 2003 \{published data only\}}

Strupp M, Schuler O, Krafczyk S, Jahn K, Schautzer F, Buttner U, et al. Treatment of downbeat nystagmus with 3,4-diaminopyridine: a placebo-controlled study. Neurology 2003;61(2):165-70.

\section{Thiagarajan 2014 \{published data only\}}

Thiagarajan P, Ciuffreda KJ, Capo-Aponte JE, Ludlam DP, Kapoor N. Oculomotor neurorehabilitation for reading in mild traumatic brain injury (mTBI): an integrative approach. Neuro Rehabilitation 2014;34(1):129-46.

\section{References to studies excluded from this review}

\section{Barton 1994 \{published data only\}}

Barton JJS, Huaman AG, Sharpe JA. Muscarinic antagonists in the treatment of acquired pendular and downbeat nystagmus: a double-blind, randomised trial of three intravenous drugs. Annals of Neurology 1994;35(3):319-25.

\section{Cifu 2014 \{published data only\}}

Cifu DX, Hoke KW, Wetzel PA, Wares JR, Gitchel G, Carne W. Effects of hyperbaric oxygen on eye tracking abnormalities in males after mild traumatic brain injury. Journal of Rehabilitation Research and Development 2014;51(7):1047-56.

\section{Claassen 2013 \{published data only\}}

Claassen J, Spiegel R, Kalla R, Faldon M, Kennard C, Danchaivijitr C, et al. A randomised double-blind, cross-over trial of 4-aminopyridine for downbeat nystagmus - effects on slowphase eye velocity, postural stability, locomotion and symptoms. Journal of Neurology, Neurosurgery and Psychiatry 2013;84(12):1392-9.

\section{Clement 2007 \{published data only\}}

Clement G, Deguine O, Bourg M, Pavy-LeTraon A. Effects of vestibular training on motion sickness, nystagmus and subjective vertical. Journal of Vestibular Research 2007;17(5-6):227-37.
Dai 2003 \{published data only\}

Dai M, Kunin M, Raphan T, Cohen B. The relation of motion sickness to the spatial-temporal properties of velocity storage. Experimental Brain Research 2003;151(2):173-89.

Feil 2013 \{published data only\}

Feil K, Claassen J, Bardins S, Teufel J, Krafczyk S, Schneider E, et al. Effect of chlorzoxazone in patients with downbeat nystagmus. Neurology 2013;81(13):1152-8.

Gur 1992 \{published data only\}

Gur S, Ron S. Training in oculomotor tracking: occupational health aspects. Israel Journal of Medical Science 1992;28(8-9):622-8.

\section{Leigh 1991 \{published data only\}}

Leigh RJ, Burnstine TH, Ruff RL, Kasmer RJ. Effect of anticholinergic agents upon acquired nystagmus:A doubleblind study of trihexyphenidyl and tridihexethyl chloride. Neurology 1991;41(11):1737-41.

\section{Leivo 1996 \{published data only\}}

Leivo S, Hernesniemi J, Luukkonen M, Vapalahti M. Early surgery improves the cure of aneurysm-induced oculomotor palsy. Surgical Neurology 1996;45(5):430-4.

Lorenz 2006 \{published data only\}

Lorenz D, Hagen K, Ufer M, Cascorbi I, Deuschl G, Volkmann J. No benefit of 3,4-diaminopyridine in essential tremor: a placebo-controlled cross-over study. Neurology 2006;66(11):1753-5.

\section{Metz 1988 \{published data only\}}

Metz HS, Mazow M. Botulinum toxin treatment of acute sixth and third nerve palsy. Graefe's Archives of Clinical and Experimental Ophthalmology 1988;226(2):141-4.

\section{Sharpe 2005 \{published data only\}}

Sharpe JA. Palsies and repair of gaze: some lessons in ocular motor learning. Neuro-Ophthalmology Japan 2005;22:125-45.

Strupp 2008 \{published data only\}

Strupp M, Kalla R, Glasauer S, Wagner J, Hufner K, Jahn K, et al. Aminopyridines for the treatment of cerebellar and ocular motor disorders. Progress in Brain Research 2008;171:535-41.

\section{Zampieri 2009 \{published data only\}}

Zampieri C, DiFabio RP. Improvement of gaze control after balance $d$ eye movement training in patients with progressive supranuclear palsy: a quasi-randomised controlled trial. Archives of Physical and Medical Rehabilitation 2009;90(2):263-70. 


\section{Additional references}

\section{Choudhuri 2007}

Choudhuri I, Sarvananthan N, Gottlob I. Survey of management of acquired nystagmus in the United Kingdom. Eye 2007;21(9):1194-7.

\section{Ciuffreda 2007}

Ciuffreda KJ, Kapoor N, Rutner D, Suchoff IB, Han ME, Craig S. Occurrence of oculomotor dysfunctions in acquired brain injury: a retrospective analysis. Optometry 2007;78(4):155-61.

\section{Craig 2008}

Craig P, Dieppe P, Maclntyre S, Michie S, Nazareth I, Petticrew M. Developing and evaluating complex interventions: new guidance. Medical Research Council 2008.

\section{Deeks 2011}

Deeks JJ, Higgins JP, Altman DG editor(s). Chapter 9: Analysing data and undertaking meta-analyses.In: Higgins JP, Green S, editor(s). Cochrane Handbook for Systematic Reviews of Interventions Version 5.1.0 (updated March 2011). The Cochrane Collaboration, 2011. Available from handbook.cochrane.org.

\section{Fowler 1996}

Fowler MS, Wade DT, Richardson AJ, Stein JF. Squints and diplopia seen after brain damage. Neurology 1996;243(1):86-90.

\section{Freeman 1988}

Freeman CF, Rudge NB. Cerebrovascular accident and the orthoptist. British Orthoptic Journal 1988;45:8-18.

\section{Glanville 2006}

Glanville JM, Lefebvre C, Miles JN, Camosso-Stefinovic J. How to identify randomized controlled trials in MEDLINE: ten years on. Journal of the Medical Library Association 2006;94(2):130-6.

\section{GRADEpro 2014 [Computer program]}

GRADE Working Group, McMaster University. GRADEpro GDT. Version accessed 12 February 2017. Hamilton (ON): GRADE Working Group, McMaster University, 2014.

\section{Gunton 2012}

Gunton KB, Brown A. Prism use in adult diplopia. Current Opinion in Ophthalmology 2012;23(5):400-4.

\section{Guyatt 2008}

Guyatt GH, Oxman AD, Vist GE, Kunz R, Falck-Ytter Y, AlonsoCoello $P$, et al. GRADE: an emerging consensus on rating quality of evidence and strength of recommendations. BMJ 2008;336:924-6.

\section{Haller 2014}

Haller T, Furr BA. Fresnel prism use among orthoptists. American Orthoptic Journal 2014;64:71-5.

\section{Hepworth 2016}

Hepworth L, Rowe FJ. Visual impairment following stroke - the impact on quality of life: a systematic review. Ophthalmology Research: an International Journal 2016;5(2):1-15.

\section{Higgins 2011a}

Higgins JP, Deeks JJ editor(s). Chapter 7: Selecting studies and collecting data. In: Higgins JP, Green S, editor(s). Cochrane Handbook for Systematic Reviews of Interventions Version 5.1.0 (updated March 2011). The Cochrane Collaboration, 2011. Available from handbook.cochrane.org.

\section{Higgins 2011b}

Higgins JP, Altman DG, Sterne JAC, editor(s). Chapter 8: Assessing risk of bias in included studies. In: Higgins JP, Green $\mathrm{S}$, editor(s). Cochrane Handbook for Systematic Reviews of Interventions Version 5.1.0 (updated March 2011). The Cochrane Collaboration, 2011. Available from handbook.cochrane.org.

\section{Higgins 2011c}

Higgins JP, Deeks JJ, Altman DG editor(s). Chapter 16: Special topics in statistics. In: Higgins JP, Green S, editor(s). Cochrane Handbook for Systematic Reviews of Interventions Version 5.1.0 (updated March 2011). The Cochrane Collaboration, 2011. Available from handbook.cochrane.org.

\section{Issaho 2017}

Issaho DC, Carvalho FR, Tabuse MK, Carrijo-Carvalho LC, de Freitas $D$. The use of botulinum toxin to treat infantile esotropia: a systematic review with meta-analysis. Investigative Ophthalmology and Visual Science 2017;58(12):5468-76.

\section{Jones 2006}

Jones SA, Shinton RA. Improving outcome in stroke patients with visual problems. Age and Ageing 2006;35(6):560-5.

\section{Kerkhoff 1999}

Kerkhoff G. Restorative and compensatory therapy approaches in cerebral blindness - a review. Restorative Neurology and Neuroscience 1999;15(2-3):255-71.

\section{Kerkhoff 2000}

Kerkhoff G. Neurovisual rehabilitation: recent developments and future directions. Journal of Neurology, Neurosurgery and Psychiatry 2000;68(6):691-706.

\section{MacIntosh 2003}

MacIntosh C. Stroke re-visited: visual problems following stroke and their effect on rehabilitation. British Orthoptic Journal 2003;60:10-4

\section{Mahan 2017}

Mahan M, Engel JM. The resurgence of botulinum toxin injection for strabismus in children. Current Opinion in Ophthalmology 2017;28(5):460-4.

\section{Moher 2009}

Moher D, Liberati A, Tetzlaff J, Altman DG, PRISMA Group. Preferred reporting items for systematic reviews and meta-analyses: the PRISMA statement. PLoS Medicine 2009;6(7):e1000097.

\section{Murad 2017}

Murad MH, Mustafa RA, Schünemann HJ, Sultan S, Santesso N. Rating the certainty in evidence in the absence of a single estimate of effect. Evidence Based Medicine 2017;22(3):85-87. 


\section{Pedersen 1981}

Pedersen RA, Troost BT. Abnormalities of gaze in cerebrovascular disease. Stroke 1981;12(2):251-4.

\section{Pierrot-Deseilligny 2011}

Pierrot-Deseilligny C. Nuclear, internuclear, and supranuclear ocular motor disorders. Handbook of Clinical Neurology 2011;102:319-31.

\section{Pigassou 1972}

Pigassou R. The functional treatment of strabismus. Canadian Journal of Ophthalmology 1972;7(3):331-5.

\section{Pollock 2011}

Pollock A, Hazelton C, Henderson CA, Angilley J, Dhillon B, Langhorne $\mathrm{P}$, et al. Interventions for disorders of eye movement in patients with stroke. Cochrane Database of Systematic Reviews 2011, Issue 10. [DOI: 10.1002/14651858.CD008389.pub2]

\section{Review Manager 2014 [Computer program]}

Nordic Cochrane Centre, The Cochrane Collaboration. Review Manager 5 (RevMan 5). Version 5.3. Copenhagen: Nordic Cochrane Centre, The Cochrane Collaboration, 2014.

\section{Rowe 2003}

Rowe FJ. Supranuclear and internuclear control of eye movements. A review. British Orthoptic Journal 2003;60:2-9.

\section{Rowe 2008}

Rowe FJ, VIS Group UK. The spectrum of nystagmus following cerebro-vascular accident. British and Irish Orthoptic Journal 2008;5:22-5.

\section{Rowe 2009}

Rowe FJ, Brand D, Jackson CA, Price A, Walker L, Harrison S, et al. Visual impairment following stroke: do stroke patients require vision assessment?. Age and Ageing 2009;38(2):188-93.

\section{Rowe 2010}

Rowe FJ, VIS Group UK. The profile of strabismus in stroke survivors. Eye 2010;24(4):682-5.

\section{Rowe 2011a}

Rowe FJ, Wright D, Brand D, Jackson C, Price A, Walker L, et al. Reading impairment following stroke: ocular and non ocular causes. International Journal of Stroke 2011;6(5):404-11.

\section{Rowe 2011b}

Rowe FJ, VIS Group UK. Prevalence of ocular motor cranial nerve palsies and associations following stroke. Eye 2011;25(7):881-7.

\section{Rowe 2013a}

Rowe FJ, Wright D, Brand D, Jackson C, Harrison S, Maan T, et al. Profile of gaze dysfunction following cerebrovascular accident. ISRN Ophthalmology 2013;2013:264604. [DOI: 10.1155/2013/264604]

\section{Rowe 2013b}

Rowe FJ, VIS Group UK. Symptoms of stroke related visual impairment. Strabismus 2013;21(2):150-4.

\section{Thurtell 2010}

Thurtell MJ, Leigh J. Therapy for nystagmus. Journal of NeuroOphthalmology 2010;30(4):361-71.

\section{References to other published versions of this review \\ Rowe 2014}

Rowe FJ, Noonan CP, Garcia-Finana M, Dodridge CS, Howard C, Jarvis KA, et al. Interventions for eye movement disorders due to acquired brain injury. Cochrane Database of Systematic Reviews 2014, Issue 9. [DOI: 10.1002/14651858.CD011290]

\section{CHARACTERISTICS OF STUDIES}

Characteristics of included studies [ordered by study ID]

Averbuch-Heller 1997

Pharmacological interventions for acquired pendular and jerk nystagmus
Allocation: double-masked
Masking: double-masked
Exclusions: 0
Losses: 1
Design: cross-over RCT

Participants

Country: 4 sites in USA and Germany

Number of participants randomised: 21 (15 with pendular nystagmus and 6 with jerk nystagmus)

Age: $25-73$ years 
Averbuch-Heller 1997 (Continued)

Gender: 10 female, 11 male

Aetiologies: multiple sclerosis (9), degeneration (1), cerebellar atrophy (1), stroke (5), idiopathic (2), encephalitis (1), tonsillar herniation (1), AIDS (1)

Ocular motility condition: acquired pendular nystagmus and horizontal jerk nystagmus

Inclusion criteria: adult nystagmus

Exclusion criteria: not specified

Interventions

Intervention 1: gabapentin

Dose: $300 \mathrm{mg}$ up to $900 \mathrm{mg} /$ day

Intervention 2: baclofen

Dose: $10 \mathrm{mg}$ up to $30 \mathrm{mg} /$ day

Duration: 2 weeks of intervention, 1-2 weeks for wash-out period, 2 weeks of intervention

\begin{tabular}{|c|c|c|}
\hline \multirow[t]{6}{*}{ Outcomes } & \multicolumn{2}{|l|}{ Measurements: } \\
\hline & \multicolumn{2}{|c|}{ Landolt C, eye movement recordings, perceived motion of target, drug effects by participant recall } \\
\hline & \multicolumn{2}{|l|}{ Timepoints: } \\
\hline & \multicolumn{2}{|c|}{ Baseline, 2 weeks, 4 weeks and 6 weeks } \\
\hline & \multicolumn{2}{|l|}{ Adverse events: } \\
\hline & \multicolumn{2}{|c|}{ Drug intolerance, Increased ataxia } \\
\hline \multirow[t]{6}{*}{ Notes } & \multicolumn{2}{|c|}{ Health economic costs: not reported } \\
\hline & \multicolumn{2}{|c|}{ Quality of life measures: not reported } \\
\hline & \multicolumn{2}{|c|}{$\begin{array}{l}\text { Funding: USPHS grant E706717, Office of Research and development, Medical research Service, De- } \\
\text { partment of Veteran Affairs and Evenon Arlington Fund and Deutsche Forschungsgemeinschaft }\end{array}$} \\
\hline & \multicolumn{2}{|c|}{$\begin{array}{l}\text { Declaration of interests: Parke-Davis Co provided transport and participant insurance fees in Ger- } \\
\text { many }\end{array}$} \\
\hline & \multicolumn{2}{|c|}{ Dates of study: not specified } \\
\hline & \multicolumn{2}{|c|}{ Trial registration ID: not specified } \\
\hline \multicolumn{3}{|l|}{ Risk of bias } \\
\hline Bias & Authors' judgement & Support for judgement \\
\hline $\begin{array}{l}\text { Random sequence genera- } \\
\text { tion (selection bias) }\end{array}$ & Unclear risk & Not specified \\
\hline \multirow[t]{2}{*}{$\begin{array}{l}\text { Allocation concealment } \\
\text { (selection bias) }\end{array}$} & Low risk & $\begin{array}{l}\text { Quote: "Patients were randomly assigned to [gabapentin or baclofen]; these } \\
\text { drugs were administered in opaque capsules that were identical in appear- } \\
\text { ance, and both the primary investigators and the patients were blinded as to } \\
\text { their identify". }\end{array}$ \\
\hline & & $\begin{array}{l}\text { Judgment comment: primary investigators were masked which suggests that } \\
\text { the allocation was concealed. }\end{array}$ \\
\hline
\end{tabular}


Averbuch-Heller 1997 (Continued)
Blinding of participants Low risk and personnel (perfor-
Quote: "Patients were randomly assigned to [gabapentin or baclofen]; these mance bias)
All outcomes drugs were administered in opaque capsules that were identical in appear- ance, and both the primary investigators and the patients were blinded as to their identify".

\begin{tabular}{lll}
\hline $\begin{array}{l}\text { Blinding of outcome as- } \\
\text { sessment (detection bias) }\end{array}$ & Low risk & $\begin{array}{l}\text { Quote: "Patients were randomly assigned to [gabapentin or baclofen]; these } \\
\text { drugs were administered in opaque capsules that were identical in appear- } \\
\text { all outcomes }\end{array}$ \\
& $\begin{array}{l}\text { ance, and both the primary investigators and the patients were blinded as to } \\
\text { their identify". }\end{array}$
\end{tabular}

\begin{tabular}{|c|c|c|}
\hline $\begin{array}{l}\text { Incomplete outcome data } \\
\text { (attrition bias) }\end{array}$ & Low risk & $\begin{array}{l}21 \text { people were recruited and } 20 \text { completed both } 2 \text {-week test periods. The one } \\
\text { person who dropped out did so because of an unrelated condition. }\end{array}$ \\
\hline
\end{tabular}

All outcomes person who dropped out did so because of an unrelated condition.

Selective reporting (re- Unclear risk No access to study protocol or trials registry entry.
porting bias)

Other bias High risk Additional 'Risk of bias' assessment for cross-over study

Was the cross-over design suitable: probably

Was there a carry-over effect: uncertain, no analysis done.

Was only first period data available: no, first period data were not available

Was the analysis correct: unclear, no estimates of effect reported

Comparability of results with those from parallel-group trials: no parallel group trials.

Kalla 2011

Pharmacological interventions for acquired downbeat nystagmus
Allocation: double-masked
Masking: double-masked
Exclusions: 0
Losses: 0
Design: cross-over RCT

Participants

\author{
Country: Germany
}

Number of participants randomised: 8

Age: mean 68 years $\pm 5.93,58-76$ years

Gender: 6 females, 2 males

Aetiologies: degeneration (2), Arnold-Chiari malformation (1), cryptogenic cerebellar ataxia (4), inflammation (1)

Ocular motility condition: downbeat nystagmus

Inclusion criteria: adult nystagmus

Exclusion criteria: not specified 
Kalla 2011 (Continued)
Interventions
Intervention 1: 4-aminopyridine

Dose: $10 \mathrm{mg}$

Intervention 2: 3,4-diaminopyridine

Dose: $10 \mathrm{mg}$

Duration: 1 day for intervention with 6-day wash-out period between interventions

\begin{tabular}{ll} 
Outcomes & Measurements: \\
3D video-oculography, drug effects by participant recall \\
Adverse events: \\
Mild paraesthesia \\
\hline Hotes \\
Qualth economic costs: not reported \\
Funding: German Ministry of Education and Research \\
Declaration of interests: authors declare no conflicts of interest \\
Dates of study: not specified \\
Trial registration ID: not specified
\end{tabular}

\section{Risk of bias}

Bias Authors' judgement Support for judgement

Random sequence genera- Unclear risk Quote: "Patients were randomly assigned capsules of 10 mg of 3,4-DAP or 4tion (selection bias) AP; they received 1 single capsule of either substance. There was a washout period of 6 days when no medication was given. One week later, the treatment was switched (i.e., they received a single capsule of the other substance)."

Judgement comment: it was not reported how the allocation sequence was generated.

$\begin{array}{ll}\begin{array}{l}\text { Allocation concealment } \\ \text { (selection bias) }\end{array} & \text { Low risk } \\ & \begin{array}{l}\text { Judgement comment: study was described as "double-blind" and identical } \\ \text { single } 10 \mathrm{mg} \text { doses used so we judge it was likely that the allocation was con- } \\ \text { cealed. }\end{array}\end{array}$

Blinding of participants Low risk
and personnel (perforQuote: "...identical single 10-mg doses of both aminopyridines were compared mance bias) in our double-blind study with crossover design"

All outcomes Judgement comment: although this information was only provided in the dis-
cussion section of the article we judge that masking of participants was likely
to have been done with identical tablets.

\begin{tabular}{|c|c|c|}
\hline $\begin{array}{l}\text { Blinding of outcome as- } \\
\text { sessment (detection bias) }\end{array}$ & Low risk & $\begin{array}{l}\text { Quote: "...identical single } 10-\mathrm{mg} \text { doses of both aminopyridines were compared } \\
\text { in our double-blind study with crossover design" }\end{array}$ \\
\hline Hall outcomes & & $\begin{array}{l}\text { Judgement comment: although this information was only provided in the dis- } \\
\text { cussion section of the article we judge that masking of outcome assessors was } \\
\text { likely to have been done with identical tablets and description of the study as } \\
\text { double-masked. }\end{array}$ \\
\hline
\end{tabular}


Kalla 2011 (Continued)

$\begin{array}{ll}\text { Incomplete outcome data Low risk } & \text { Judgement comment: the article describes a study of } 8 \text { patients. Loss to fol- } \\ \text { (attrition bias) } & \text { low-up was not mentioned. }\end{array}$

All outcomes

low-up was not mentioned.

Selective reporting (re- Unclear risk No access to study protocol or trials registry entry. porting bias)

Other bias High risk Additional 'Risk of bias' assessment for cross-over study

Was the cross-over design suitable: probably

Was there a carry-over effect: uncertain, no analysis done.

Was only first period data are available: no, first period data not available

Was the analysis correct: unclear, no estimates of effect reported

Comparability of results with those from parallel-group trials: no parallel group trials

Methods Botulinum toxin versus observation of acute onset sixth nerve palsy

Allocation: random number table

Masking: not achieved

Exclusions: 2 due to change in diagnosis

Losses: 5 lost to follow-up

Study design: parallel RCT

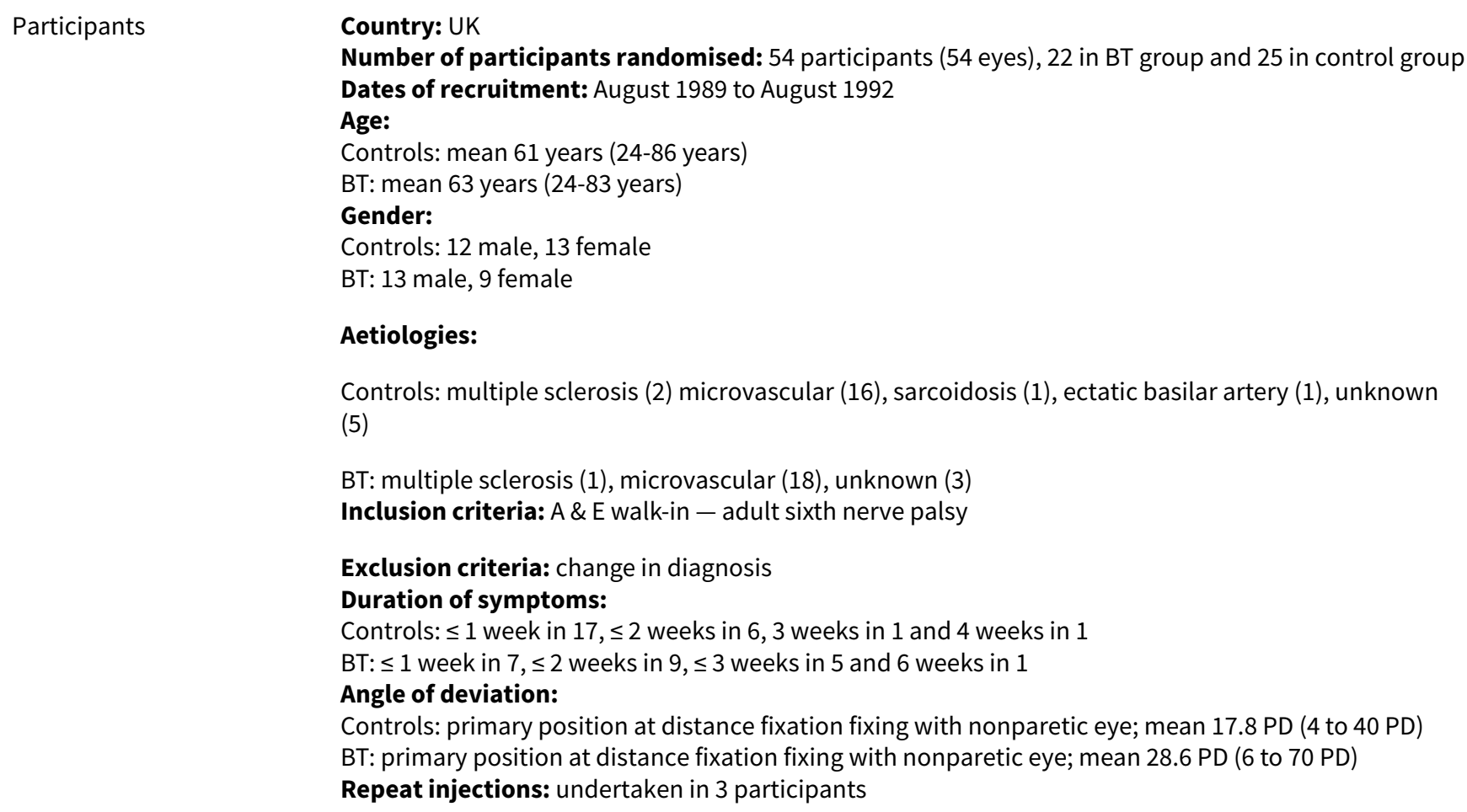


Lee 1994 (Continued)

BT: 2.5 units Dysport ${ }^{\text {TM }}$ to ipsilateral medial rectus muscle. 3 participants had a second injection when first injection was inadequate

Control: observation

Duration: 4-42 months. Participants were followed up at 1 week, 6 weeks and 4 months as a minimum and were discharged at 4 months if fully recovered.

Choice of eye for intervention: ipsilateral eye to the cranial nerve palsy - conventional choice

\begin{tabular}{ll}
\hline Outcomes & Measurements: \\
& Ocular motility range - abduction deficit. Binary response of yes/no. Angle of deviation by prism cover \\
test. Field of binocular single vision. Participant-reported symptoms. Adverse reactions.
\end{tabular}

Notes

Health economic costs: not reported

Quality of life measures: not reported

Funding: None specified

Declarations of interest: None specified

Trial registration number: None specified

\section{Risk of bias}

\begin{tabular}{lll}
\hline Bias & Authors' judgement & Support for judgement \\
\hline $\begin{array}{l}\text { Random sequence genera- } \\
\text { tion (selection bias) }\end{array}$ & Low risk & $\begin{array}{l}\text { Quote "...patients were randomly assigned to "treatment" or "control" groups } \\
\text { by reference to a random number table." }\end{array}$ \\
\hline $\begin{array}{l}\text { Allocation concealment } \\
\text { (selection bias) }\end{array}$ & High risk & $\begin{array}{l}\text { Judgement comment: as the treatments were quite different - botulinum toxin } \\
\text { versus observation - it is likely that the Investigators were aware of participant } \\
\text { allocation. }\end{array}$ \\
\hline $\begin{array}{l}\text { Blinding of participants } \\
\text { and personnel (perfor- } \\
\text { mance bias) }\end{array}$ & High risk & $\begin{array}{l}\text { Judgement comment: as the treatments were quite different - botulinum tox- } \\
\text { in versus observation - it is likely that the participants and their carers were } \\
\text { aware of participant allocation. }\end{array}$
\end{tabular}

\begin{tabular}{|c|c|c|}
\hline $\begin{array}{l}\text { Blinding of outcome as- } \\
\text { sessment (detection bias) } \\
\text { All outcomes }\end{array}$ & High risk & $\begin{array}{l}\text { Judgement comment: as the treatments were quite different - botulinum toxin } \\
\text { versus observation - it is likely that the outcome assessors were aware of par- } \\
\text { ticipant allocation. }\end{array}$ \\
\hline
\end{tabular}

Incomplete outcome data Unclear risk (attrition bias)

Quote "Of the initial 54 patients, five (four controls, one injection) were lost to All outcomes follow-up. A further two patients (both controls) were later excluded because of a change in diagnosis."

Judgement comment: follow-up was clearly described and most participants were followed-up $(47 / 54,87 \%)$. However, loss to follow-up appeared to occur predominantly in the control group. It is unclear what impact that would have had.

\begin{tabular}{lll}
\hline $\begin{array}{l}\text { Selective reporting (re- } \\
\text { porting bias) }\end{array}$ & Unclear risk & No access to study protocol or trials registry entry. \\
\hline Other bias & Unclear risk & Not applicable \\
\hline
\end{tabular}

\section{Strupp 2003}

\section{Methods}

Pharmacological interventions for acquired downbeat nystagmus 
Allocation: double-masked

Masking: double-masked

Exclusions: 1 (chronic alcohol use)

Losses: 0

Design: cross-over RCT

Participants

Country: Germany

Dates of recruitment: March 2002 to September 2002

Number of participants randomised: 18

Age: $50-85$ years

Gender: 9 female, 9 male

Aetiologies: Arnold-Chiari malformation (1), degeneration (4), cerebellar ataxia (1), stroke (3), unknown (8)

Ocular motility condition: acquired downbeat nystagmus

Inclusion criteria: pure downbeat nystagmus, downbeat nystagmus with associated central vestibular or ocular motility disorders

Exclusion criteria: epileptic seizures, cardiac arrhythmia, taking drugs affecting the central nervous system or vestibular system

Interventions

Intervention: 3,4-diaminopyridine

Dose: $20 \mathrm{mg}$

Control: lactose placebo

Duration: 1 day of intervention, 1-2 weeks for wash-out period, 1 day of control

\section{Measurements:}

2-dimensional video-oculography, perceived motion of target, drug effects by participant recall

Timepoints:

Baseline, 1 day and 2 weeks

\section{Adverse events:}

Transient minor perioral or digital paraesthesia, nausea

Health economic costs: not reported

Quality of life measures: not reported

Funding: not specified

Declaration of interests: not specified

Trial registration ID: not specified

\section{Risk of bias}


Strupp 2003 (Continued)

Random sequence genera- Low risk Quote: "With use of a computer-generated randomization list" tion (selection bias)

\begin{tabular}{|c|c|c|}
\hline $\begin{array}{l}\text { Allocation concealment } \\
\text { (selection bias) }\end{array}$ & Low risk & $\begin{array}{l}\text { Quote: "Code envelopes were kept by the investigator during the trial and re- } \\
\text { turned unopened to the monitor after termination of the study. The blind was } \\
\text { maintained until data analysis had been completed." }\end{array}$ \\
\hline
\end{tabular}

\begin{tabular}{ll}
$\begin{array}{l}\text { Blinding of participants } \\
\text { and personnel (perfor- }\end{array}$ & Quote: "Capsules with 20 mg of 3,4-DAP and lactose or placebo (a capsule with \\
mance bias) & $\begin{array}{l}\text { lactose alone) were manufactured and delivered by the pharmacy of the Uni- } \\
\text { versity of Munich (Klinikum Grosshadern). The shape and color of the capsules } \\
\text { All outcomes }\end{array}$ \\
\hline
\end{tabular}

\begin{tabular}{ll}
\hline $\begin{array}{l}\text { Blinding of outcome as- } \\
\text { sessment (detection bias) }\end{array}$ & Low risk \\
All outcomes & $\begin{array}{l}\text { Quote: "Capsules with } 20 \text { mg of 3,4-DAP and lactose or placebo (a capsule with } \\
\text { lactose alone) were manufactured and delivered by the pharmacy of the Uni- } \\
\text { versity of Munich (Klinikum Grosshadern). The shape and color of the capsules } \\
\text { with 3,4-DAP or placebo were identical." } \\
\text { Quote: "Code envelopes were kept by the investigator during the trial and re- } \\
\text { turned unopened to the monitor after termination of the study. The blind was } \\
\text { maintained until data analysis had been completed." }\end{array}$ \\
\hline
\end{tabular}

$\begin{array}{ll}\begin{array}{l}\text { Incomplete outcome data } \\ \text { (attrition bias) }\end{array} & \text { Low risk } \\ \text { All outcomes } & \begin{array}{l}\text { Quote: "Seventeen patients (nine men; aged } 50 \text { to } 85 \text { years) with DBN were in- } \\ \text { cluded in the study; one patient had to be excluded because of chronic alcohol } \\ \text { consumption even on the day of the planned examination" }\end{array} \\ & \begin{array}{l}\text { Judgement comment: this excluded participant appeared to be excluded be- } \\ \text { fore randomisation. All } 17 \text { participants completed the study. }\end{array}\end{array}$

\begin{tabular}{|c|c|c|}
\hline $\begin{array}{l}\text { Selective reporting (re- } \\
\text { porting bias) }\end{array}$ & Unclear risk & No access to study protocol or trials registry entry. \\
\hline
\end{tabular}

Other bias High risk Additional 'Risk of bias' assessment for cross-over study

Was the cross-over design suitable: probably

Was there a carry-over effect: uncertain, no analysis done.

Was only first period data are available: no, first period data not available

Was the analysis correct: unclear, no estimates of effect reported

Comparability of results with those from parallel-group trials: no parallel group trials

\begin{tabular}{ll}
\hline Methods & Oculomotor rehabilitation versus sham training for traumatic brain injury \\
Allocation: single masked \\
Masking: single masked \\
Exclusions: 0 \\
Losses: 0 \\
Design: cross-over RCT \\
\hline Participants
\end{tabular}


Thiagarajan 2014 (Continued)

\section{Number of participants randomised: 12}

Age: $29 \pm 3$ years

Gender: not specified

Aetiologies: type of acquired brain injury not specified

Ocular motility condition: any acquired disorder

Inclusion criteria: TBI onset at least one year post-incident to ensure that any subsequent changes during training are not secondary to their natural neurological recovery function period (6-9 months). Participants exhibit at least one symptom (e.g. skipping lines while reading, blur, diplopia, etc.) and one clinical sign (e.g. receded near point of convergence) of a non-strabismic oculomotor dysfunction related to impaired sustained reading. Intact cognitive ability to perform the required tasks for the study. Stable systemic health.

Exclusion criteria: persons over the age of 40 years, as they typically will not have sufficient accommodation to measure reliably. Best corrected visual acuity poorer than 20/30 in either eye. Constant strabismus, amblyopia, or ocular disease in either eye. Medications that alter oculomotor function or attentional state (or both)

Intervention: ocular motor rehabilitation - training of versions, vergence and accommodation for 15 minutes each interspaced with 5 minute rest intervals.

Dose: 2 sessions of 60 minutes training per week, block of 6 weeks

Control: sham treatment of basic reading tasks

Dose: 2 sessions of 60 minutes training per week, block of 6 weeks

Duration: 2 blocks of 6 weeks with one-week interim wash-out

\section{Outcomes Measurements:}

Reading rate, infra-red eye recording of reading eye movements, saccade ratio - progression and regression saccades by eye movement recording, binocular accommodative amplitude, near point of convergence, convergence insufficiency symptom survey questionnaire

\section{Timepoints:}

Baseline, 6 weeks and post final block

Notes Health economic costs: not reported

Funding: US Army, DoD award, College of Optometrists in Vision Development and SUNY Graduate programme

Dates of study: not reported

Declaration of interests: no interests to declare

Trial registration ID: not specified

\section{Risk of bias}

\begin{tabular}{lll}
\hline Bias & Authors' judgement & Support for judgement \\
\hline $\begin{array}{l}\text { Random sequence genera- } \\
\text { tion (selection bias) }\end{array}$ & Low risk & $\begin{array}{l}\text { Quote: "During phase 1, every odd-numbered subject first received OMT, and } \\
\text { every even-numbered subject first received ST, and vice-versa during phase 2." }\end{array}$ \\
\hline $\begin{array}{l}\text { Allocation concealment } \\
\text { (selection bias) }\end{array}$ & High risk & Single-masked \\
\hline
\end{tabular}


Thiagarajan 2014 (Continued)

Blinding of participants Unclear risk Quote: "A cross-over, interventional experimental design of a single-blinded and personnel (performance bias)

All outcomes

\author{
nature (for the subject) was used."
}

Judgement comment: this implies the participants were masked to the intervention, but the intervention and control are so different it is likely that the participants may be influenced by knowledge of the intervention. It is unclear what the impact of this would have been and may be considered to be part of the intervention so we have graded this as unclear risk of bias.

Blinding of outcome as- High risk Not masked

sessment (detection bias)

All outcomes

\begin{tabular}{lll}
\hline $\begin{array}{l}\text { Incomplete outcome data } \\
\text { (attrition bias) } \\
\text { All outcomes }\end{array}$ & Low risk & \\
\hline $\begin{array}{l}\text { Selective reporting (re- } \\
\text { porting bias) }\end{array}$ & Unclear risk & No access to study protocol or trials registry entry. \\
\hline Other bias & High risk & Additional risk of bias assessment for cross-over study \\
& Was the cross-over design suitable: probably not \\
& $\begin{array}{l}\text { Was there a carry-over effect: uncertain, no analysis done. } \\
\text { Was only first period data are available: no, first period data not available } \\
\text { Was the analysis correct: unclear, no estimates of effect reported, data for in- } \\
\text { tervention group only reported } \\
\text { Comparability of results with those from parallel-group trials: no parallel } \\
\text { group trials }\end{array}$ \\
\hline
\end{tabular}

A \& E: Accident and Emergency

AIDS: acquired immune deficiency syndrome

BT: botulinum toxin

PD: prism dioptre

$\mathrm{RCT}$ : randomised controlled trial

SEM: standard error mean

TBI: traumatic brain injury

USPHS: United States Public Health Service

Characteristics of excluded studies [ordered by study ID]

\begin{tabular}{ll}
\hline Study & Reason for exclusion \\
\hline Barton 1994 & $\begin{array}{l}\text { Six of seven cases were either multiple sclerosis or degenerative; only one case of potential ac- } \\
\text { quired brain injury }\end{array}$ \\
\hline Cifu 2014 & Not a randomised controlled trial \\
\hline Claassen 2013 & $\begin{array}{l}25 \text { of } 27 \text { cases were either idiopathic or degenerative; only two cases of potential acquired brain in- } \\
\text { jury }\end{array}$ \\
\hline Clement 2007 & Cases were normal participants with induced vestibular imbalance; no acquired brain injury \\
\hline Dai 2003 & Cases were normal participants with induced imbalance; no acquired brain injury \\
\hline \hline
\end{tabular}




\begin{tabular}{ll}
\hline Study & Reason for exclusion \\
\hline Feil 2013 & No cases of acquired brain injury; all participants were idiopathic or degenerative \\
\hline Gur 1992 & Not a randomised controlled trial \\
\hline Leigh 1991 & $\begin{array}{l}\text { Seven of ten cases were either multiple sclerosis or degenerative; only three cases of acquired } \\
\text { brain injury }\end{array}$ \\
\hline Leivo 1996 & Not a randomised controlled trial \\
\hline Lorenz 2006 & No cases of acquired brain injury; all with essential tremor \\
\hline Metz 1988 & Not a randomised controlled trial \\
\hline Sharpe 2005 & Not a randomised controlled trial \\
\hline Strupp 2008 & Not a randomised controlled trial \\
\hline Zampieri 2009 & No cases of acquired brain injury; all with progressive supranuclear palsy \\
\hline
\end{tabular}

\section{ADDITIONAL TABLES}

\section{Table 1. Botulinum toxin versus observation}

\begin{tabular}{|c|c|c|c|c|c|}
\hline Study ID & Total participants & $\begin{array}{l}\text { Primary: im- } \\
\text { proved ocular } \\
\text { motility }\end{array}$ & $\begin{array}{l}\text { Secondary: improved binocular sin- } \\
\text { gle vision }\end{array}$ & $\begin{array}{l}\text { Secondary: im- } \\
\text { proved symp- } \\
\text { toms }\end{array}$ & $\begin{array}{l}\text { Secondary: } \\
\text { adverse } \\
\text { events }\end{array}$ \\
\hline Lee 1994 & $\begin{array}{l}47 \text {, parallel arm } \\
\mathrm{RCT} \\
22 \text { - botulinum } \\
\text { toxin } \\
25 \text { - observation } \\
6 \text { month follow-up }\end{array}$ & $\begin{array}{l}21(95.5 \%) \text { - } \\
\text { botulinum tox- } \\
\text { in } \\
20(80 \%) \text { - ob- } \\
\text { servation }\end{array}$ & $\begin{array}{l}\text { Success: } \\
21(95.5 \%) \text { - botulinum toxin } \\
20(80 \%) \text { - observation } \\
\text { Partial: } \\
3(12 \%) \text { - observation } \\
\text { Fail: } \\
1(4.5 \%) \text { - botulinum toxin } \\
2(8 \%) \text { - observation }\end{array}$ & $\begin{array}{l}21(95.5 \%) \text { - } \\
\text { botulinum tox- } \\
\text { in } \\
20(80 \%) \text { - ob- } \\
\text { servation }\end{array}$ & $\begin{array}{l}9 \% \text { ptosis } \\
18 \% \text { vertical } \\
\text { deviation }\end{array}$ \\
\hline
\end{tabular}

$\mathrm{RCT}$ : randomised controlled trial

\section{Table 2. Oculomotor rehabilitation versus sham training}

\begin{tabular}{|c|c|c|c|c|c|}
\hline Study ID & $\begin{array}{l}\text { Total partici- } \\
\text { pants }\end{array}$ & $\begin{array}{l}\text { Primary: improved } \\
\text { ocular motility }\end{array}$ & Secondary: improved functional vision & $\begin{array}{l}\text { Secondary: } \\
\text { improved } \\
\text { symptoms }\end{array}$ & $\begin{array}{l}\text { Secondary: } \\
\text { adverse } \\
\text { events }\end{array}$ \\
\hline $\begin{array}{l}\text { Thiagarajan } \\
2014\end{array}$ & $\begin{array}{l}\text { 12, cross-over } \\
\mathrm{RCT}\end{array}$ & $\begin{array}{l}\text { Baseline } 2.1 \text { sac- } \\
\text { cadic ratio reduc- } \\
\text { ing to } 1.7, \mathrm{P}<0.05\end{array}$ & Reading rate: & $\begin{array}{l}\text { Improved for } \\
\text { OM rehabilita- } \\
\text { tion. }\end{array}$ & Nil reported \\
\hline
\end{tabular}


Table 2. Oculomotor rehabilitation versus sham training (Continued)

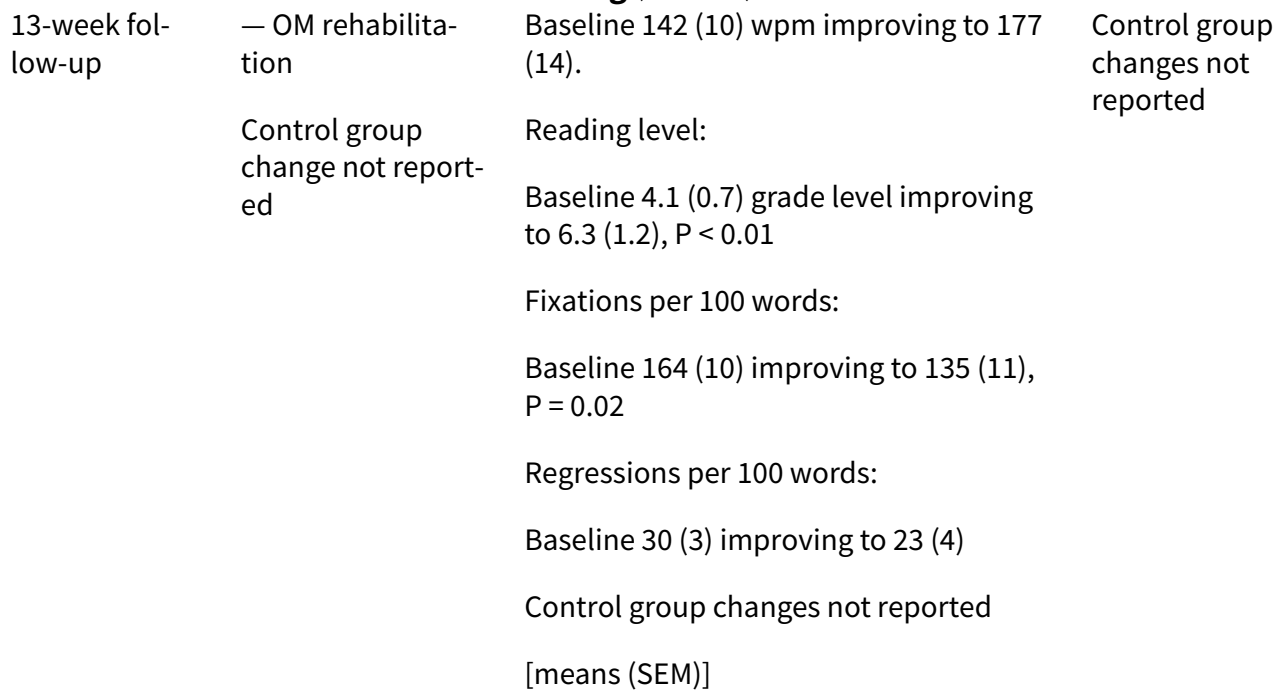

SEM: standard error mean

OM: oculo motor

$\mathrm{RCT}$ : randomised controlled trial

WPM: words per minute

\section{Table 3. Pharmacological treatment for nystagmus}

\begin{tabular}{|c|c|c|c|c|c|}
\hline Study ID & Total participants & $\begin{array}{l}\text { Primary: improved ocular } \\
\text { motility }\end{array}$ & $\begin{array}{l}\text { Secondary: } \\
\text { improved vi- } \\
\text { sual acuity }\end{array}$ & $\begin{array}{l}\text { Secondary: im- } \\
\text { proved symp- } \\
\text { toms }\end{array}$ & $\begin{array}{l}\text { Secondary: adverse } \\
\text { events }\end{array}$ \\
\hline \multirow[t]{4}{*}{$\begin{array}{l}\text { Aver- } \\
\text { buch-Heller } \\
1997\end{array}$} & $\begin{array}{l}21, \text { crossover RCT } \\
15 \text { - pendular }\end{array}$ & \multirow[t]{4}{*}{15 pendular - gabapentin } & $\begin{array}{l}15 \text { pendular - } \\
\text { gabapentin }\end{array}$ & $\begin{array}{l}6 \text { pendular - } \\
\text { gabapentin }\end{array}$ & $\begin{array}{l}1 \text { drug intolerance - } \\
\text { gabapentin }\end{array}$ \\
\hline & 6 - jerk & & $\begin{array}{l}1 \text { jerk - } \\
\text { gabapentin }\end{array}$ & $\begin{array}{l}1 \text { jerk - } \\
\text { gabapentin }\end{array}$ & $\begin{array}{l}4 \text { drug intolerance - } \\
\text { baclofen }\end{array}$ \\
\hline & \multirow[t]{2}{*}{$\begin{array}{l}\text { 6-week trial dura- } \\
\text { tion }\end{array}$} & & \multirow[t]{2}{*}{$\begin{array}{l}1 \text { jerk - ba- } \\
\text { clofen }\end{array}$} & \multirow[t]{2}{*}{1 jerk - baclofen } & 3 ataxia - gabapentin \\
\hline & & & & & 2 ataxia - baclofen \\
\hline \multirow[t]{2}{*}{ Kalla 2011} & $\begin{array}{l}\text { 8, crossover RCT } \\
8 \text { - downbeat }\end{array}$ & $\begin{array}{l}\text { Baseline }-6.04 \text {; } 45 \text { mins }-1.58 \text {; } \\
90 \text { mins }-1.21 \text { (4-aminopyri- } \\
\text { dine) }\end{array}$ & \multirow[t]{2}{*}{-} & \multirow[t]{2}{*}{-} & \multirow[t]{2}{*}{$\begin{array}{l}\text { All with mild paraes- } \\
\text { thesia }\end{array}$} \\
\hline & $\begin{array}{l}\text { 8-day trial dura- } \\
\text { tion }\end{array}$ & $\begin{array}{l}\text { Baseline - } 5.68 \text {; } 45 \text { mins }-3.29 \text {; } \\
90 \text { mins - } 2.96 \text { (3,4-diaminopy- } \\
\text { ridine) }\end{array}$ & & & \\
\hline \multirow[t]{2}{*}{ Strupp 2003} & $\begin{array}{l}17 \text {, crossover RCT } \\
17 \text { - downbeat }\end{array}$ & $\begin{array}{l}\text { Baseline } 7.2 \pm 4.2 \% \text { sec reduc- } \\
\text { ing to } 3.1 \pm 2.5 \text { ( } 3,4 \text {-diaminopy- } \\
\text { ridine) }\end{array}$ & - & \multirow{2}{*}{$\begin{array}{l}10 \text { - reduced } \\
\text { symptoms (3,4- } \\
\text { diaminopyri- } \\
\text { dine) } \\
0 \text { - reduced } \\
\text { symptoms } \\
\text { (placebo) }\end{array}$} & $\begin{array}{l}3 \text { - mild paraesthesia } \\
\text { (3,4-diaminopyridine) }\end{array}$ \\
\hline & $\begin{array}{l}\text { 16-day trial dura- } \\
\text { tion }\end{array}$ & $\begin{array}{l}\text { Baseline } 7.4 \pm 4.1 \% \text { sec reduc- } \\
\text { ing to } 7.3 \pm 3.7 \text { (placebo) }\end{array}$ & & & $\begin{array}{l}1 \text { - nausea/headache } \\
\text { (3,4-diaminopyridine) }\end{array}$ \\
\hline
\end{tabular}

$\mathrm{RCT}$ : randomised controlled trial 


\section{AP PE N DICES}

\section{Appendix 1. CENTRAL search strategy}

\#1 MeSH descriptor: [Brain Injuries] explode all trees

\#2 brain near/2 injur*

$\# 3 \mathrm{ABI}$ or TBI or non TB

\#4 \#1 or \#2 or \#3

\#5 MeSH descriptor: [Eye] explode all trees

\#6 MeSH descriptor: [Visually Impaired Persons] explode all trees

\#7 MeSH descriptor: [Ocular Physiological Processes] explode all trees

\#8 MeSH descriptor: [Diagnostic Techniques, Ophthalmological] explode all trees

\#9 MeSH descriptor: [Optometry] explode all trees

\#10 MeSH descriptor: [Orthoptics] explode all trees

\#11 MeSH descriptor: [Eye Diseases] this term only

\#12 MeSH descriptor: [Vision Disorders] this term only

\#13 MeSH descriptor: [Eye Manifestations] this term only

\#14 MeSH descriptor: [Blindness] this term only

\#15 MeSH descriptor: [Diplopia] explode all trees

\#16 MeSH descriptor: [Vision, Binocular] this term only

\#17 MeSH descriptor: [Vision, Monocular] this term only

\#18 MeSH descriptor: [Visual Acuity] explode all trees

\#19 MeSH descriptor: [Visual Fields] this term only

\#20 MeSH descriptor: [Vision, Low] this term only

\#21 MeSH descriptor: [Visual Field Tests] explode all trees

\#22 MeSH descriptor: [Ophthalmology] this term only

\#23 MeSH descriptor: [Vision Screening] this term only

\#24 MeSH descriptor: [Eye Diseases, Hereditary] explode all trees

\#25 MeSH descriptor: [Ocular Motility Disorders] explode all trees

\#26 MeSH descriptor: [Optic Nerve Diseases] explode all trees

\#27 MeSH descriptor: [Orbital Diseases] explode all trees

\#28 MeSH descriptor: [Pupil Disorders] explode all trees

\#29 MeSH descriptor: [Refractive Errors] explode all trees

\#30 MeSH descriptor: [Blindness, Cortical] explode all trees

\#31 MeSH descriptor: [Hemianopsia] explode all trees

\#32 MeSH descriptor: [Scotoma] this term only

\#33 MeSH descriptor: [Abducens Nerve] this term only

\#34 MeSH descriptor: [Oculomotor Nerve] this term only

\#35 MeSH descriptor: [Trochlear Nerve] this term only

\#36 "smooth pursuit" or saccades or saccadic or "depth perception" or stereopsis or gaze disorder* or ophthalm* or optic nerve*

\#37 (downbeat or upbeat or vertical) near/3 nystagmus

\#38 gaze* near/2 (deficit* or pals* or disorder ${ }^{\star}$ )

\#39 ocular near/2 (muscle* or align*)

\#40 esotropi* or exotropi* or hypertropi ${ }^{\star}$ or hypotropi* or cyclotropi ${ }^{\star}$

\#41 intranuclear ophthalmoplegia or parinaud's syndrome or weber's syndrome or skew deviation or conjugate deviation

\#42 (visual ${ }^{\star}$ or vision or eye or eyes or eyesight or sight) near/3 (problem or disorder $^{\star}$ or impair ${ }^{\star}$ or disabilit* or loss or disease* or defect* or manifestation* or screening or test* or examination $\left.{ }^{\star}\right)$

\#43 reading near/2 (difficult* or impair ${ }^{\star}$ )

\#44 hemianop* or blindness or low vision or refractive errors or scotoma or diplopia or optometr* or ocular or orthoptic*

\#45 oscillopsia or visual tracking or fresnel prism*

\#46 III or IV or VI or third or fourth or sixth near/3 nerve palsy

$\# 47$ (\#5 or \#6 or \#7 or \#8 or \#9 or \#10 or \#11 or \#12 or \#13 or \#14 or \#15 or \#16 or \#17 or \#18 or \#19 or \#20 or \#22 or \#23 or \#24 or \#25 or \#26 or \#27 or \#28 or \#29 or \#30 or \#31 or \#32 or \#33 or \#34 or \#35 or \#36 or \#41 or \#42 or \#44 or \#45 or \#46)

\#48 \#4 and \#47

\section{Appendix 2. MEDLINE Ovid search strategy}

1. randomized controlled trial.pt.

2. (randomized or randomised).ab,ti.

3. placebo.ab,ti. 
4. dt.fs.

5. randomly.ab,ti.

6. trial.ab,ti.

7. groups.ab,ti.

8. or/1-7

9. exp animals/

10. exp humans/

11.9 not (9 and 10)

12. 8 not 11

13. exp brain injuries/

14. (brain adj2 injur\$).tw.

15. (ABI or TBI or non $\mathrm{TBI})$.tw.

16. or $/ 13-15$

17. exp Eye/

18. exp Visually Impaired Persons/

19. exp Ocular Physiological Processes/

20. exp Diagnostic Techniques, Ophthalmological/

21. exp Optometry/

22. exp Orthoptics/

23. exp Eye Diseases/

24. exp Vision Disorders/

25. exp Eye Manifestations/

26. exp Blindness/

27. exp Diplopia/

28. Vision, Binocular/

29. Vision, Monocular/

30. exp Visual Acuity/

31. Visual Fields/

32. Vision, Low/

33. exp Visual Field Tests/

34. Ophthalmology/

35. Vision Screening/

36. Eye Diseases, Hereditary/

37. exp Ocular Motility Disorders/

38. exp Optic Nerve Diseases/

39. Enophthalmos/

40. exp Pupil Disorders/

41. exp Refractive Errors/

42. Blindness, Cortical/

43. exp Hemianopsia/

44. Scotoma/

45. Abducens Nerve/

46. Oculomotor Nerve/

47. Trochlear Nerve/

48. (smooth pursuit or saccades or saccadic or depth perception or stereopsis or gaze disorder\$ or ophthalm\$ or optic nerve\$).tw.

49. (ocular adj2 (muscle\$ or align\$)).tw.

50. (esotropi\$ or exotropi\$ or hypertropi\$ or hypotropi\$ or cyclotropi\$).tw.

51. (intranuclear ophthalmoplegia or parinaud's syndrome or weber's syndrome or skew deviation or conjugate deviation).tw.

52. ((visual\$ or vision or eye or eyes or eyesight or sight) adj3 (problem\$ or disorder\$ or impair\$ or disabilit\$ or loss or disease\$ or defect $\$$ or manifestation\$ or screening or test\$ or examination\$)).tw.

53. (reading adj2 (difficult\$ or impair\$)).tw.

54. (hemianop\$ or blindness or low vision or refractive errors or scotoma or diplopia or optometr\$ or ocular or orthoptic\$).tw.

55. (oscillopsia or visual tracking or fresnel prism\$).tw.

56. or/17-55

57. 12 and 16 and 56

58. ((downbeat or upbeat or vertical) adj3 nystagmus).tw.

59. (gaze\$ adj2 (deficit\$ or pals\$ or disorder\$)).tw.

60. ((III or IV or VI or third or fourth or sixth) adj3 nerve pals\$).tw.

61. or/58-60

62. 12 and 61

63.57 or 62 
The search filter for trials at the beginning of the MEDLINE strategy is from the published paper by Glanville 2006.

\section{Appendix 3. Embase Ovid search strategy}

1. exp randomized controlled trial/

2. exp randomization/

3. exp double blind procedure/

4. exp single blind procedure/

5. random\$.tw.

6. or/1-5

7. (animal or animal experiment).sh.

8. human.sh.

9.7 and 8

10.7 not 9

11.6 not 10

12. exp clinical trial/

13. (clin\$ adj3 trial\$).tw.

14. ((singl\$ or doubl\$ or trebl\$ or tripl\$) adj3 (blind\$ or mask\$)).tw.

15. exp placebo/

16. placebo\$.tw.

17. random\$.tw.

18. exp experimental design/

19. exp crossover procedure/

20. exp control group/

21. exp latin square design/

22. or $/ 12-21$

23. 22 not 10

24. 23 not 11

25. exp comparative study/

26. exp evaluation/

27. exp prospective study/

28. (control\$ or prospectiv\$ or volunteer\$).tw.

29. or $/ 25-28$

30.29 not 10

31.30 not (11 or 23 )

32. 11 or 24 or 31

33. exp brain injury/

34. (brain adj2 injur\$).tw.

35. (ABI or TBI or non $\mathrm{TBI})$.tw.

36. or/33-35

37. eye/

38. visual system function/

39. visual system examination/

40. optometry/

41. orthoptics/

42. eye disease/

43. visual disorder/

44. exp eye movement disorder/

45. exp visual impairment/

46. exp vision/

47. perimetry/

48. ophthalmology/

49. exp optic nerve disease/

50. exp cranial nerve/

51. (smooth pursuit or saccades or saccadic or depth perception or stereopsis or gaze disorder\$ or ophthalm\$ or optic nerve\$).tw.

52. (ocular adj2 (muscle\$ or align\$)).tw.

53. (esotropi\$ or exotropi\$ or hypertropi\$ or hypotropi\$ or cyclotropi\$).tw.

54. (intranuclear ophthalmoplegia or parinaud's syndrome or weber's syndrome or skew deviation or conjugate deviation).tw.

55. ((visual\$ or vision or eye or eyes or eyesight or sight) adj3 (problem $\$$ or disorder $\$$ or impair $\$$ or disabilit $\$$ or loss or disease $\$$ or defect $\$$ or manifestation $\$$ or screening or test\$ or examination\$)).tw.

56. (reading adj2 (difficult\$ or impair\$)).tw.

57. (hemianop\$ or blindness or low vision or refractive errors or scotoma or diplopia or optometr\$ or ocular or orthoptic\$).tw. 
58. (oscillopsia or visual tracking or fresnel prism\$).tw.

59. or $/ 37-58$

60.32 and 36 and 59

61. ((downbeat or upbeat or vertical) adj3 nystagmus).tw.

62. (gaze\$ adj2 (deficit\$ or pals\$ or disorder\$)).tw.

63. ((III or IV or VI or third or fourth or sixth) adj3 nerve pals\$).tw.

64. or/61-63

65.32 and 64

66.60 or 65

\section{Appendix 4. CINAHL EBSCO search strategy}

\section{S56 S12 AND S55}

S55 S16 AND S54

$\mathrm{S} 54 \mathrm{~S} 51$ or $\mathrm{S} 52$ or $\mathrm{S} 53$

$\mathrm{S} 53 \mathrm{~S} 41$ or $\mathrm{S} 42$ or $\mathrm{S} 43$ or $\mathrm{S} 44$ or $\mathrm{S} 45$ or $\mathrm{S} 46$ or $\mathrm{S} 47$ or $\mathrm{S} 48$ or $\mathrm{S} 49$ or $\mathrm{S} 50$ or S51

$\mathrm{S} 52 \mathrm{~S} 31$ or $\mathrm{S} 32$ or $\mathrm{S} 33$ or $\mathrm{S} 34$ or $\mathrm{S} 35$ or $\mathrm{S} 36$ or $\mathrm{S} 37$ or $\mathrm{S} 38$ or $\mathrm{S} 39$ or $\mathrm{S} 40$

$\mathrm{S} 51 \mathrm{~S} 17$ or S18 or S19 S20 or S21 or S22 or S23 or S24 or S25 or S26 or S27 or S28 or S29 or S30

S50 nerve palsy

S49 oscillopsia or visual tracking or fresnel prism*

S48 hemianop* or blindness or low vision or refractive errors or scotoma or diplopia or optometr* or ocular or orthoptic*

S47 reading N2 (difficult* or impair ${ }^{\star}$ )

S46 (visual ${ }^{\star}$ or vision or eye or eyes or eyesight or sight) N5 (problem* or disorder ${ }^{\star}$ or impair ${ }^{\star}$ or disabilit ${ }^{\star}$ or loss or disease $^{\star}$ or defect $^{\star}$ or manifestation* or screening or test* or examination*)

S45 intranuclear ophthalmoplegia or parinaud's syndrome or weber's syndrome or skew deviation or conjugate deviation

S44 esotropi* or exotropi ${ }^{\star}$ or hypertropi ${ }^{\star}$ or hypotropi* or cyclotropi ${ }^{\star}$

S43 ocular N2 (muscle* or align*)

S42 gaze $^{\star}$ N2 (deficit* or palsy or disorder ${ }^{\star}$ )

S41 nystagmus or smooth pursuit or saccades or depth perception or stereopsis or ophthalmol ${ }^{\star}$ or optic nerve

S40 (MH "Trochlear Nerve") OR (MH "Trochlear Nerve Diseases")

S39 (MH "Abducens Nerve Diseases+") OR (MM "Abducens Nerve")

S38 (MM "Refractive Errors+")

S37 (MH "Pupil Disorders+")

S36 (MH "Orbital Diseases+")

S35 (MH "Optic Nerve Diseases+")

S34 (MM "Ocular Motility Disorders")

S33 (MM "Eye Diseases, Hereditary")

S32 (MM "Ophthalmology")

S31 (MH "Vision Tests+")

S30 (MH "Visual Perception+")

S29 (MM "Visual Fields")

S28 (MH "Visual Acuity")

S27 (MH "Depth Perception")

S26 (MH "Vision, Subnormal")

S25 (MM "Diplopia")

S24 (MH "Blindness+")

S23 (MH "Eye Manifestations+")

S22 (MH "Vision Disorders+")

S21 (MH "Eye Diseases+")

S20 (MM "Optometry")

S19 (MH "Diagnosis, Eye+")

S18 (MH "Rehabilitation of Vision Impaired+")

S17 (MH "Eye+")

$\mathrm{S} 16 \mathrm{~S} 13$ or S14 or S15

$\mathrm{S} 15 \mathrm{ABI}$ or TBI or non TBI

S14 brain N2 injur*

S13 (MH "Brain Injuries+")

$\mathrm{S} 12 \mathrm{~S} 1$ or S2 or S3 or S4 or S5 or S6 or S7 or S8 or S9 or S10 or S11

S11 TX allocat ${ }^{\star}$ random ${ }^{\star}$

S10 (MM "Quantitative Studies")

S9 (MM "Placebos")

S8 TX placebo* 
S7 TX random* allocat* S6 (MM "Random Assignment")

S5 TX randomi* control $^{*}$ trial $^{\star}$

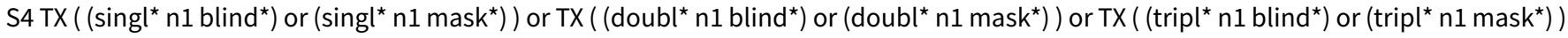

or TX ( (trebl* $n 1$ blind $\left.{ }^{\star}\right)$ or (trebl* $n 1$ mask $\left.^{\star}\right)$ )

S3 TX clinic $^{\star}$ n1 trial ${ }^{\star}$

S2 PT Clinical trial

S1 (MH "Clinical Trials+")

\section{Appendix 5. AMED Ovid search strategy}

1. "Randomized controlled trials"/

2. prospective studies/

3. single blind method/

4. random\$.tw.

5. placebo\$.tw.

6. trial\$.tw.

7. groups.tw.

8. ((singl\$ or doubl\$) adj3 (blind\$ or mask\$)).tw.

9. or/1-8

10. brain injuries/

11. (brain adj2 injur\$).tw.

12. (ABI or TBI or non $T B I)$.tw.

13. or $/ 10-12$

14. eye/

15. Blindness/

16. Vision disorders/

17. Vision/

18. Ocular Motility Disorders/

19. Eye Movements/

20. Optic nerve/

21. Refractive Errors/

22. Cranial nerves/

23. (smooth pursuit or saccades or saccadic or depth perception or stereopsis or gaze disorder\$ or ophthalm\$ or optic nerve\$).tw.

24. (ocular adj2 (muscle\$ or align\$)).tw.

25. (esotropi\$ or exotropi\$ or hypertropi\$ or hypotropi\$ or cyclotropi\$).tw.

26. (intranuclear ophthalmoplegia or parinaud's syndrome or weber's syndrome or skew deviation or conjugate deviation).tw.

27. ((visual\$ or vision or eye or eyes or eyesight or sight) adj3 (problem\$ or disorder\$ or impair\$ or disabilit\$ or loss or disease\$ or defect

$\$$ or manifestation\$ or screening or test\$ or examination\$)).tw.

28. (reading adj2 (difficult\$ or impair\$)).tw.

29. (hemianop\$ or blindness or low vision or refractive errors or scotoma or diplopia or optometr\$ or ocular or orthoptic\$).tw.

30. (oscillopsia or visual tracking or fresnel prism\$).tw.

31. or/14-30

32. 9 and 13 and 31

33. ((downbeat or upbeat or vertical) adj3 nystagmus).tw.

34. (gaze\$ adj2 (deficit\$ or pals\$ or disorder\$)).tw.

35. ((III or IV or VI or third or fourth or sixth) adj3 nerve palsy).tw.

36. or/33-35

37.9 and 36

3832 or 37

\section{Appendix 6. PsychINFO Ovid search strategy}

1. exp Treatment Effectiveness Evaluation/

2. $\exp$ Clinical Trials/

3. exp Placebo/

4. placebo\$.tw.

5. randomly.tw.

6. randomi\#ed.tw.

7. trial\$.tw.

8. ((singl\$ or doubl\$ or trebl\$ or tripl\$) adj3 (blind\$ or mask\$ or dummy)).tw.

9. (factorial\$ or allocat\$ or assign $\$$ or volunteer $\$$ ).tw.

10. (crossover\$ or cross over\$).tw. 
11. (quasi adj (experimental or random\$)).tw.

12. (control\$ adj3 (trial\$ or study or studies or group\$)).tw.

13. or $/ 1-12$

14. exp Brain Damage/

15. exp Traumatic Brain Injury/

16. (ABI or TBI or non TB).tw.

17. exp Head Injuries/

18. or/14-17

19. exp Eye Disorders/

20. exp Eye Fixation/

21. exp "Pupil (Eye)"/

22. exp Vision Disorders/

23. exp Vision/

24. exp Blind/

25. exp Optometry/

26. exp Strabismus/

27. $\exp$ Amblyopia/

28. exp Binocular Vision/

29. exp Eye Convergence/

30. exp Color Perception/

31. exp Visual Acuity/

32. exp Visual Field/

33. exp Ophthalmology/

34. exp Ophthalmologic Examination/

35. exp Hemianopia/

36. exp Abducens Nerve/

37. exp Cranial Nerves/

38. (smooth pursuit or saccades or saccadic or depth perception or stereopsis or gaze disorder\$ or ophthalm\$ or optic nerve\$).tw.

39. (ocular adj2 (muscle\$ or align\$)).tw.

40. (esotropi\$ or exotropi\$ or hypertropi\$ or hypotropi\$ or cyclotropi\$).tw.

41. (intranuclear ophthalmoplegia or parinaud's syndrome or weber's syndrome or skew deviation or conjugate deviation).tw.

42. ((visual\$ or vision or eye or eyes or eyesight or sight) adj3 (problem $\$$ or disorder\$ or impair\$ or disabilit\$ or loss or disease $\$$ or defect

$\$$ or manifestation $\$$ or screening or test $\$$ or examination $\$)$ ).tw.

43. (reading adj2 (difficult\$ or impair\$)).tw.

44. (hemianop\$ or blindness or low vision or refractive errors or scotoma or diplopia or optometr\$ or ocular or orthoptic\$).tw.

45. (oscillopsia or visual tracking or fresnel prism\$).tw.

46. or/19-45

47. 13 and 18 and 46

48. ((downbeat or upbeat or vertical) adj3 nystagmus).tw.

49. (gaze\$ adj2 (deficit\$ or pals\$ or disorder\$)).tw.

50. ((III or IV or VI or third or fourth or sixth) adj3 nerve pals\$).tw.

51. or $/ 48-50$

52.13 and 51

53.47 or 52

\section{Appendix 7. Dissertations and Theses (PQDT) database}

Keywords (dissertation topic) $=$ brain injury

\section{Appendix 8. PsycBITE database}

PsycBITE was searched using the following options from the search interface:

Neurological Group = Traumatic Brain Injury (TBI) / Head Injury

Method $=$ Randomised Controlled Trials

Target area $=$ Literacy/Numeracy OR Visual Field Loss OR Community Re-entry

\section{Appendix 9. ISRCTN search strategy}

brain Injury

nystagmus

gaze deficit 
nerve palsy

\section{Appendix 10. ClinicalTrials.gov search strategy}

brain Injury OR nystagmus OR gaze deficit OR nerve palsy

\section{Appendix 11. Health Services Research Projects in Progress}

brain Injury

nystagmus

gaze deficit

nerve palsy

\section{Appendix 12. National Eye Institute Clinical Studies Database}

brain Injury

nystagmus

gaze deficit

nerve palsy

\section{Appendix 13. WHO ICTRP search strategy}

brain Injury

nystagmus

gaze deficit

nerve palsy

\section{Appendix 14. Standardised headings for included studies table}

Headings in table in Proposed subheadings
RevMan

\begin{tabular}{ll}
\hline Study design & Parallel group RCT, i.e. people randomised to treatment \\
& - Paired eye or intra-individual RCT, i.e. eyes randomised to treatment \\
& - Cluster RCT, i.e. communities randomised to treatment \\
& - Cross-over RCT \\
& Other
\end{tabular}

\begin{tabular}{ll}
\hline Participants & Country \\
\cline { 2 - 2 } Setting \\
\hline Number of participants \\
Number of men \\
\hline
\end{tabular}


(Continued)

Average age

Age range

Ethnic group

Inclusion criteria

Exclusion criteria

Eye movement disor- $\quad$ Description of the type of eye movement disorder (III, IV, and VI cranial nerve ders palsy, reduced fixation, gaze holding, gaze palsy, saccadic problems, smooth pursuit problems, strabismus, nystagmus, reduced convergence or divergence, conjugate deviation, skew deviation), the deviation of eye movement (horizontal, vertical, torsional), and the severity of eye movement disorder (slight, small, moderate, marked; paralysis, paresis; monocular, binocular).

Acquired brain injury Description of type of brain injury, natural history, side of brain injury.

$\begin{array}{ll}\text { Interventions } & \text { We provide a description of interventions given to each treatment group in- } \\ \text { Comparator } & \begin{array}{l}\text { cluding, if relevant, the duration, intensity, frequency, or dose. We classify the } \\ \text { type of intervention as restitution, compensation, pharmacological or substi- } \\ \text { tution, type of brain injury, type of eye movement disorder, and the type of } \\ \text { control as no treatment, placebo, control, or standard care. We document the } \\ \text { professional background of the person providing the intervention (e.g. oph- } \\ \text { thalmologist, orthoptist). }\end{array}\end{array}$

Outcomes List We document the primary and secondary outcomes relevant to this review
as listed in the Types of outcome measures section. Specifically, we document measurements showing change in angle of deviation and/or extent of eye movement range, measurement of binocular single vision, documentation of participant-reported symptoms, documentation of questionnaires and adverse events. If a study has used a number of different methods of measuring the same outcome (e.g. prisms and degrees for measurement of ocular deviation), we note each method to be used for any subsequent analysis.

\begin{tabular}{ll} 
Notes & Indicating specific dates of recruitment of participants $\mathrm{mm} / \mathrm{yr}$ to $\mathrm{mm} / \mathrm{yr}$ \\
\hline \multicolumn{2}{l}{ Sources of funding } \\
\hline Declaration of interest & Indicating any declarations of interest among the primary researchers \\
\hline Other & $\begin{array}{l}\text { We record any important confounding variables. If a study includes more than } \\
\text { two intervention groups, we also record the method of including these groups } \\
\text { in any subsequent analysis. }\end{array}$ \\
\hline
\end{tabular}

\section{CONTRIBUTIONSOF AUTHORS}

FR proposed the review questions, co-ordinated the review, organised retrieval of papers and wrote to trial authors for additional information.

FR and KH screened search results, screened retrieved papers against inclusion criteria, appraised quality of papers, extracted data from papers, provided additional data about papers, obtained and screened data on unpublished studies and entered data in Review Manager.

JE provided methodological expertise. 
All authors provided additional content expertise, provided clinical, policy and consumer perspectives, read and commented on final drafts of the review.

\section{DECLARATIONSOF INTEREST}

Fiona Rowe is an National Institute of Health Research (NIHR) Research Fellow and has received funding from the NIHR to support research relating to visual problems after stroke (including this Cochrane Review).

The work presented here represents the view of the authors and not necessarily those of the funding bodies.

\section{SOURCES OF SUPPORT}

\section{Internal sources}

- No sources of support supplied

\section{External sources}

- National Institute for Health Research (NIHR), UK.

- NIHR have funded the research fellowship programme that encompasses this Review.

- Richard Wormald, Co-ordinating Editor for Cochrane Eyes and Vision (CEV) acknowledges financial support for his CEV research sessions from the Department of Health through the award made by the NIHR to Moorfields Eye Hospital NHS Foundation Trust and UCL Institute of Ophthalmology for a Specialist Biomedical Research Centre for Ophthalmology.

- This review was supported by the NIHR, via Cochrane Infrastructure funding to the CEV UK editorial base.

The views and opinions expressed therein are those of the authors and do not necessarily reflect those of the Systematic Reviews Programme, NIHR, NHS or the Department of Health.

\section{DIFFERENCES BETWEEN PROTOCOLANDREVIEW}

\section{Objectives}

During the course of doing the review it became clear that it would not make sense to pool data on the pre-specified different types of interventions (restitutive, substitutive, compensatory and pharmacological) as they are clinically so different. Our original protocol objectives specified a primary objective which implied that these interventions would be pooled, if data were available. We amended our objectives to make it clear that we considered these different groups of interventions separately.

\section{Inclusion criteria}

We clarified the following inclusion criteria.

- Type of studies: we clarified the inclusion of cross-over trials.

- Type of participants: we clarified the inclusion of studies of mixed aetiologies.

- Type of participants: we clarified the exclusion of participants with multiple sclerosis and degenerative conditions.

\section{Search strategy}

We amended the search strategy to include additional terms for nystagmus, nerve palsy and gaze disorders.

\section{Amendment to methods}

- Measures of treatment effect: we used the risk ratio rather than the odds ratio as planned, since this provides a better assessment of the treatment effect. Odds ratios are always more exaggerated (larger or smaller) and in this review there were no analytical issues that would warrant using the odds ratio.

- Assessment of risk of bias: As four out of the five included studies were cross-over studies, we amended the protocol to include additional 'Risk of bias' assessment criteria for cross-over studies (Higgins 2011c).

- GRADE and 'Summary of findings' table: these were not specified in the protocol but we added them because they have since become mandatory Cochrane methods (methods.cochrane.org/mecir).

\section{Planned methods that were not used}

- We did not undertake any meta-analyses so the following planned methods were not implemented.

- Measures of treatment effect: we specified the use of the standardised mean difference and also how we would manage change data and final value data but this was not needed. 
- Dealing with missing data: we planned to assess trials that included intention-to-treat analyses to ensure this had been done correctly, ensuring participants had been included even if they did not fully adhere to the protocol and that it was possible to extract the appropriate data for these participants from the results.

- Assessment of heterogeneity: we planned to examine the forest plots and use the Chi 2 test and 12 test to assess heterogeneity.

- Assessment of reporting bias: we planned to create a funnel plot if there were 10 or more trials in any analysis.

- Data synthesis: we planned to combine data using a random-effects model (unless there was a small number of trials in which case we would have used a fixed-effect model).

- Subgroup analysis: initially we planned to consider the different types of intervention - restitutive, compensatory, substitutive and pharmacological - as subgroup analyses, however in reality but the interventions in these categories were so different we considered that an overall meta-analysis would be unlikely to be informative (even if the data were available), so we considered these comparisons separately. Other planned subgroup analyses of gender, type of acquired brain injury, side of brain injury, type of eye movement disorder, deviation of eye movement, and severity of eye movement were also not possible.

- Sensitivity analyses: we planned sensitivity analyses to test the effect of any assumptions regarding missing data, and effects of publication type and risk of bias.

\section{Additional authors}

This review includes the authors Kerry Hanna and Jennifer Evans who were not involved in the protocol authorship.

\section{N DEX TERMS}

\section{Medical Subject Headings (MeSH)}

4-Aminopyridine [*analogs \& derivatives] [therapeutic use]; Abducens Nerve Diseases [etiology]; Amifampridine; Amines [*therapeutic use]; Baclofen [ ${ }^{\star}$ therapeutic use]; Botulinum Toxins [adverse effects] [^therapeutic use]; Brain Injuries [ ${ }^{\star}$ complications]; Cyclohexanecarboxylic Acids [ ${ }^{\star}$ therapeutic use]; Gabapentin; Neuromuscular Agents [adverse effects] [*therapeutic use]; Nystagmus, Pathologic [etiology] [therapy]; Ocular Motility Disorders [*drug therapy] [etiology]; Randomized Controlled Trials as Topic; Vision, Binocular; Watchful Waiting; gamma-Aminobutyric Acid [ ${ }^{\star}$ therapeutic use]

\section{MeSH check words}

Humans 